\title{
Lattice-Boltzmann lattice-spring simulations of two flexible fibers settling in moderate Reynolds number flows
}

\author{
Ahmed Abdulkareem Alhasan ${ }^{\mathrm{a}}$, Ye Luo ${ }^{\mathrm{a}}$, Tai-Hsien $\mathrm{Wu}^{\mathrm{a}}$, Guowei He ${ }^{\mathrm{b}}$, Dewei $\mathrm{Qi}^{\mathrm{a}}{ }^{\mathrm{a}}$ * \\ a Department of Chemical and Paper Engineering, Western Michigan University, Michigan, USA \\ ${ }^{\mathrm{b}}$ State-Key Lab for Nonlinear Mechanics, Institute of Mechanics, Chinese Academy of Sciences, China
}

\section{A R T I C L E I N F O}

\section{Article history:}

Received 17 September 2017

Revised 7 February 2018

Accepted 10 March 2018

Available online 12 March 2018

\section{Keywords:}

Lattice Boltzmann method

Lattice spring model

Hemodynamics

Elastic vessel

Blockage

\begin{abstract}
A B S T R A C T
Dynamic motion of two flexible fibers settling in an infinitely long fluid column at moderate Reynolds numbers is numerically simulated in a three-dimensional space by using a lattice-Boltzmann latticespring method. In the simulations, rectangular and cylindrical flexible fibers are used. The fiber rigidity, density, shape, initial distance, and aspect ratio are varied at different levels and their effects on drafting, kissing, and tumbling (DKT) behavior are studied. It is demonstrated that the fiber rigidity has a profound impact on settling velocity, fluid structures, and DKT phenomenon.
\end{abstract}

(c) 2018 Elsevier Ltd. All rights reserved.

\section{Introduction}

Liquid-solid particle handling systems, such as fluidized beds in reactors, screening, filtration, and sedimentation, are commonly encountered in chemical, pharmaceutical, petroleum, food process, paper, and biological engineering [1]. For example, in water treatment sedimentation is used to remove precipitants coagulated in water softeners [2]. In the paper engineering, since fines have huge surface areas, receive larger drag forces, and settle much slower than long fibers, the fines can be separated from the long fibers. The sedimentation is also used as a primary stage in modern waste water treatment plants to reduce the content of suspended solids and the pollutants embedded. Due to importance to industries, many experimental, theoretical, and computational studies are conducted to understand the mechanisms of dynamic motion of solid particles in fluid [3], either coagulation or dispersion. This may help engineers to design a fluid-solid particle handling system with a high operation efficiency.

In 1987, Fortes et. al. [4] observed experimentally that when two particles sediment vertically in a channel filled with a Newtonian fluid, the trailing particle may accelerate and approach the leading particle, called drafting, due to the suction effect of the low pressure in the wakes behind the leading particle. When the trailing particle attaches the leading particle, called kissing, the suction effect is reduced and the two particles become unstable, and they

\footnotetext{
* Corresponding author.

E-mail address: dewei.qi@wmich.edu (D. Qi).
}

exchange their positions. The trailing particle may pass the leading particle and become the leading particle, called tumbling. This drafting, kissing, and tumbling (DKT) phenomenon, closely associated with inertial effects, may repeatedly take place during settling process. Late, Feng et. al. [5] used finite element method to simulate the motion of particles settling in a vertical channel and numerically confirmed the DKT phenomenon. Further, many others [6] used different methods to simulate the particle motion and revealed that particle density, initial vertical distance, and diameter ratio have a profound influence on the DTK behavior. Recently using lattice Boltzmann simulations, Wang et. al. [6] reported that when the diameter ratio of two circular particles is smaller than 1.21 , DKT phenomena are repeated many times and when the diameter ratio is larger than 1.22 , DKT occurs only once.

A recent literature search reveals that most studies concentrated on either rigid particles or flexible particles at zero Reynolds number flows and very limited results were reported on flexible or deformable particles at moderate Reynolds numbers, where inertia cannot be ignored, although sedimentation of fibers [79] at the Stokes fluid were extensively studied. The work for flexible fibers at moderate Reynolds numbers was reported by $\mathrm{Wu}$ et. al. [10], who employed a flexible particle method, based on lattice Boltzmann method, to simulate single and multi-flexible fibers. They demonstrated that a flexible fiber has a larger settling velocity than a stiff fiber and multi-flexible fibers have significantly larger settling velocity than the corresponding isolated single fiber. However, they did not explore the DKT behavior of two flexible fibers. Therefore, the present work will employ a lattice-Boltzmann 


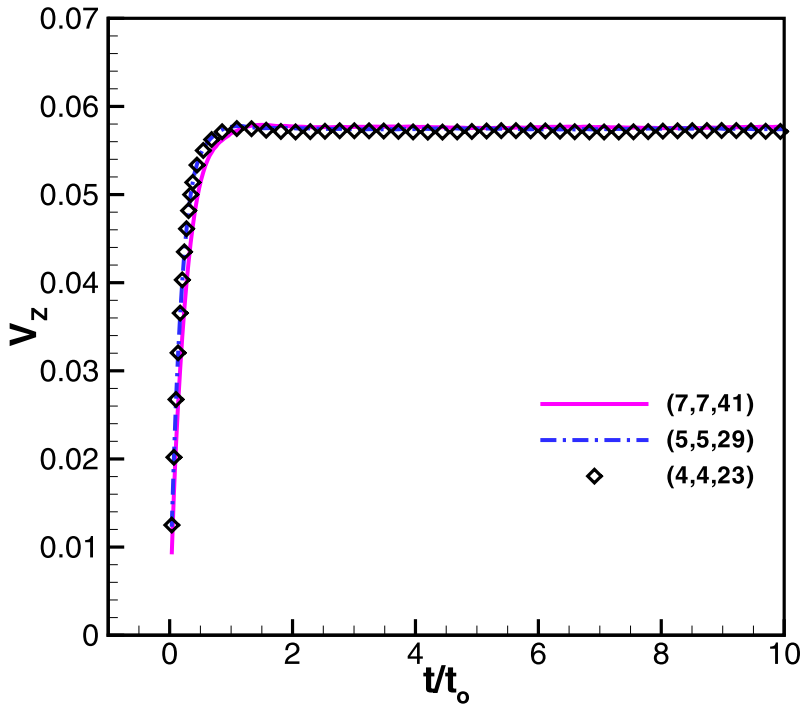

Fig. 1. The settling velocity as a function of time for the same case at three different resolutions.

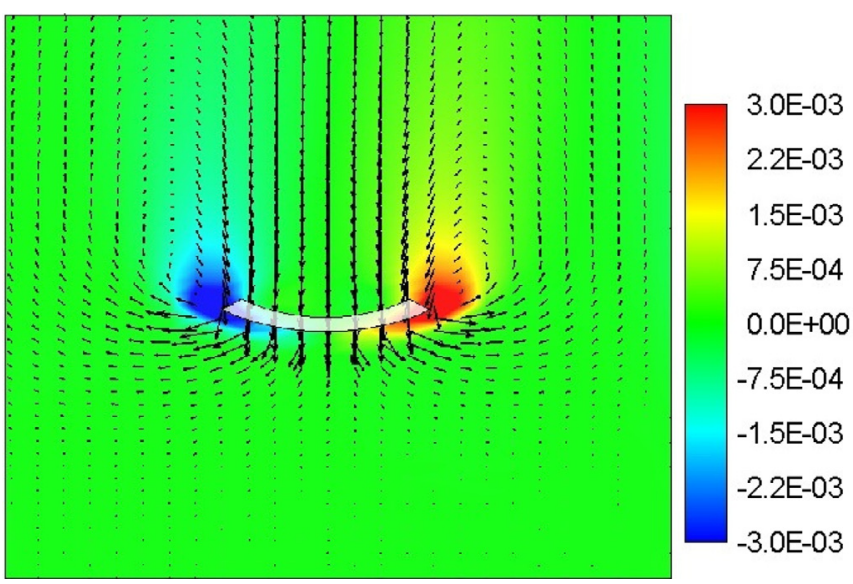

Fig. 2. The velocity fields and tip vortices around the area of the two ends of the single fiber at $E I=0.048$.

lattice-spring method to mimic DKT phenomenon of two flexible fibers settling down in an infinitely long rectangular fluid channel at moderate Reynolds numbers. In the simulations, flexibility, fiber shape, Reynolds number, shape, and, aspect ratio are varied at different levels and their effects on the DKT behavior and fluid structures are investigated.

In next section, the LBLS method is briefly introduced and a non-dimensional elasto-gravitation number is defined and used to analyze possible deformation. The simulation results of single and two flexible fibers are reported in Section 3. Effects of rigidity, aspect ratio, initial distance, fiber shape, and Reynolds number on DKT are studied. Conclusions are made in the last section.

\section{Simulation methods}

\subsection{Non-dimensionalization}

Dynamic motion of fluids follows the Navier-Stokes equations while fiber motion and deformation can be approximated by a beam motion equation. The sedimentation behavior of a fiber in fluids is mainly determined by hydrodynamic force, fiber elasticity, and gravity. These equations can be non-dimensionalized if the length, velocity, time, and force are re-scaled by the fiber length $L$,

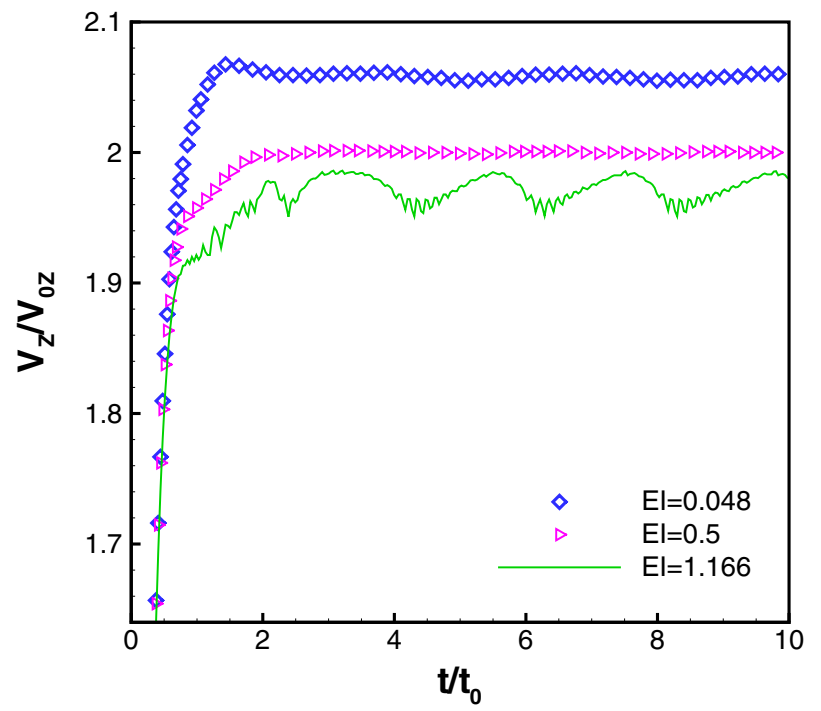

Fig. 3. Comparison of the fiber settling velocity as a function of time among three different levels of rigidity. The vertical velocity $V_{z}$ is normalized by the Stokes velocity $V_{0 z}$ and the time $t$ is normalized by $t_{0}=L / V_{0 z}$. The same applies to other figures.
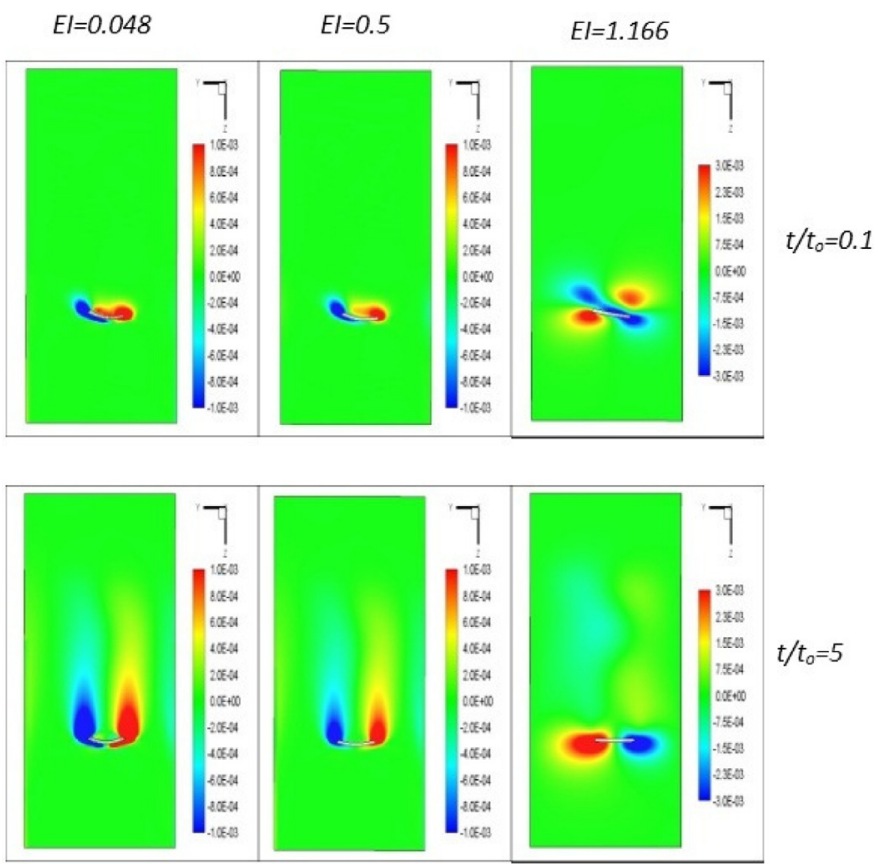

Fig. 4. Comparison of the vorticity in the X-direction in the cross section of $x=30$ among three different levels of the rigidity at two different time instants a) $t / t_{0}=$ 0.01 (top) b) $t / t_{0}=5$ (bottom).

reference velocity $V_{0}$, time $L / V_{0}$, and $\mu V_{0} L$, respectively, where $\mu$ is the viscosity. The non-dimensional Navier-Stokes equations for fluids can be written by

$\nabla \cdot \mathbf{u}=0$

$\operatorname{Re}\left(\frac{\partial \mathbf{u}}{\partial t}+\mathbf{u} \cdot \nabla \mathbf{u}\right)=-\nabla p+\nabla^{2} \mathbf{u}$,

where $R e=V_{0} L / v$ is the particle Reynolds number, $p$ is the pressure, and the non-dimensional beam equation can be written by

$E I \frac{\partial^{4} w}{\partial l^{4}}+\frac{\rho_{s}}{\rho_{f}} \frac{\partial w^{2}}{\partial t^{2}}=\frac{L^{2} F_{f}}{D B R_{s d}}+\left(\frac{\rho_{s}}{\rho_{f}}-1\right) F r^{-1}$, 


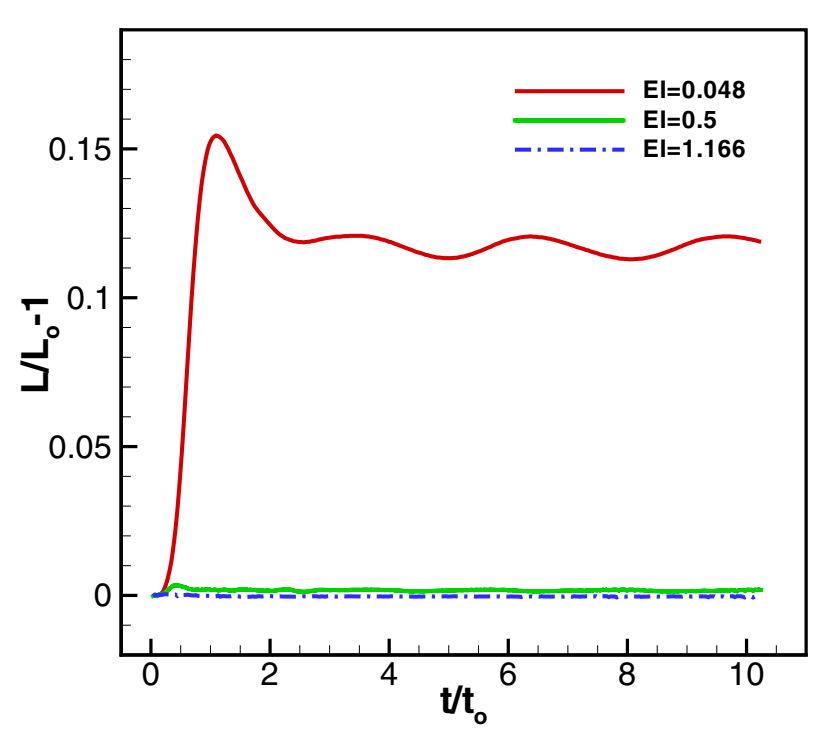

Fig. 5. Comparison of the fiber curl index as a function of time among three different levels of the rigidity.

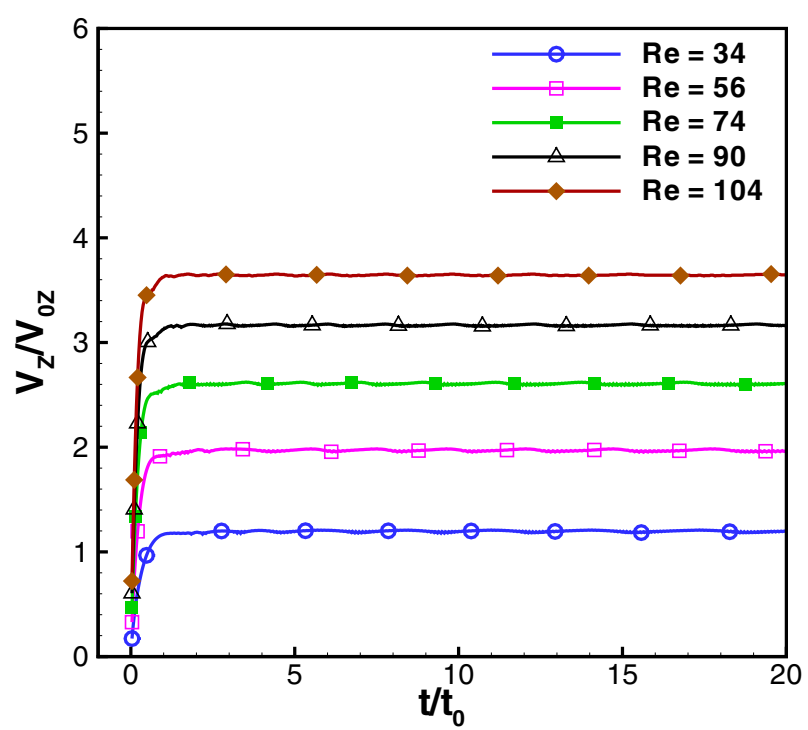

Fig. 6. Comparison of the settling velocity of a single fiber as a function of time among different Reynolds numbers for the case with the same rigidity of $E I=0.971$

a)

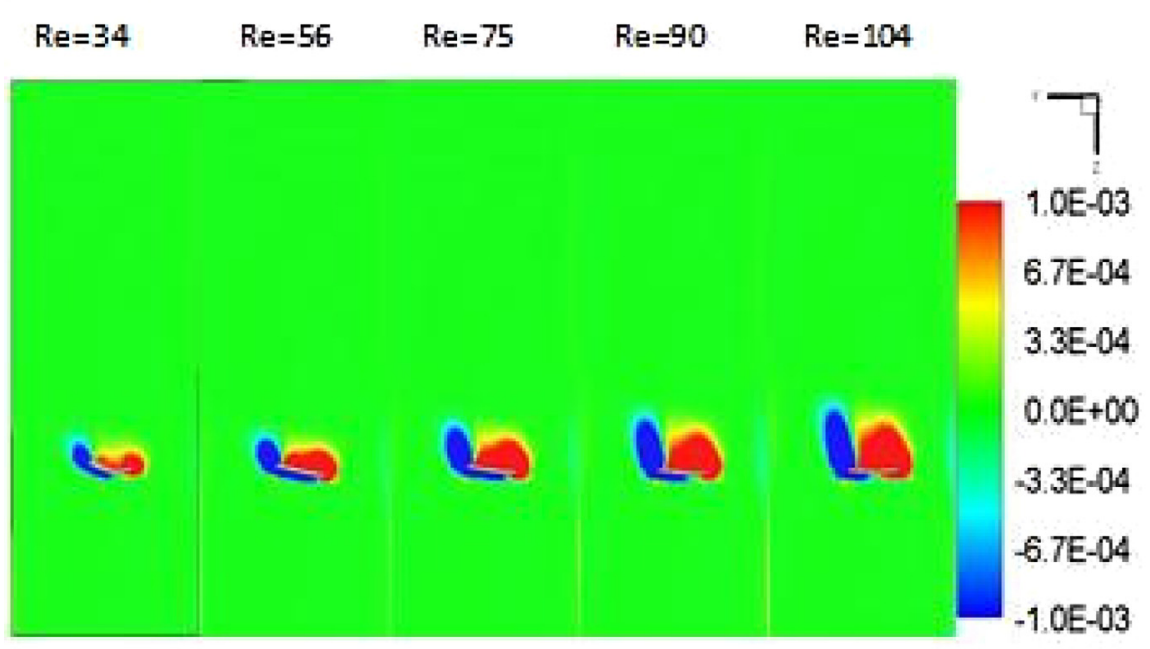

b)

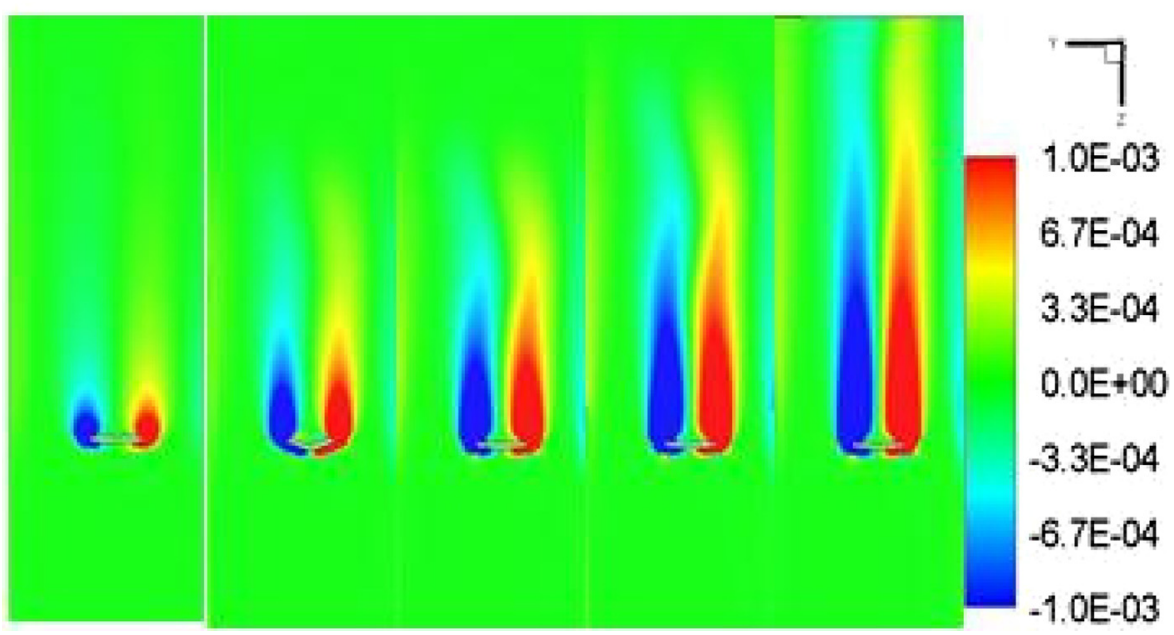

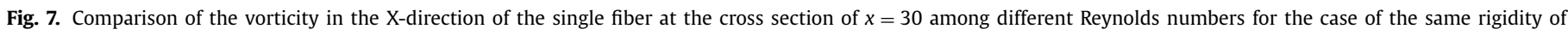
$E I=0.971$ at (a) $t / t_{0}=0.01 ;$ (b) at $t / t_{0}=5$. 

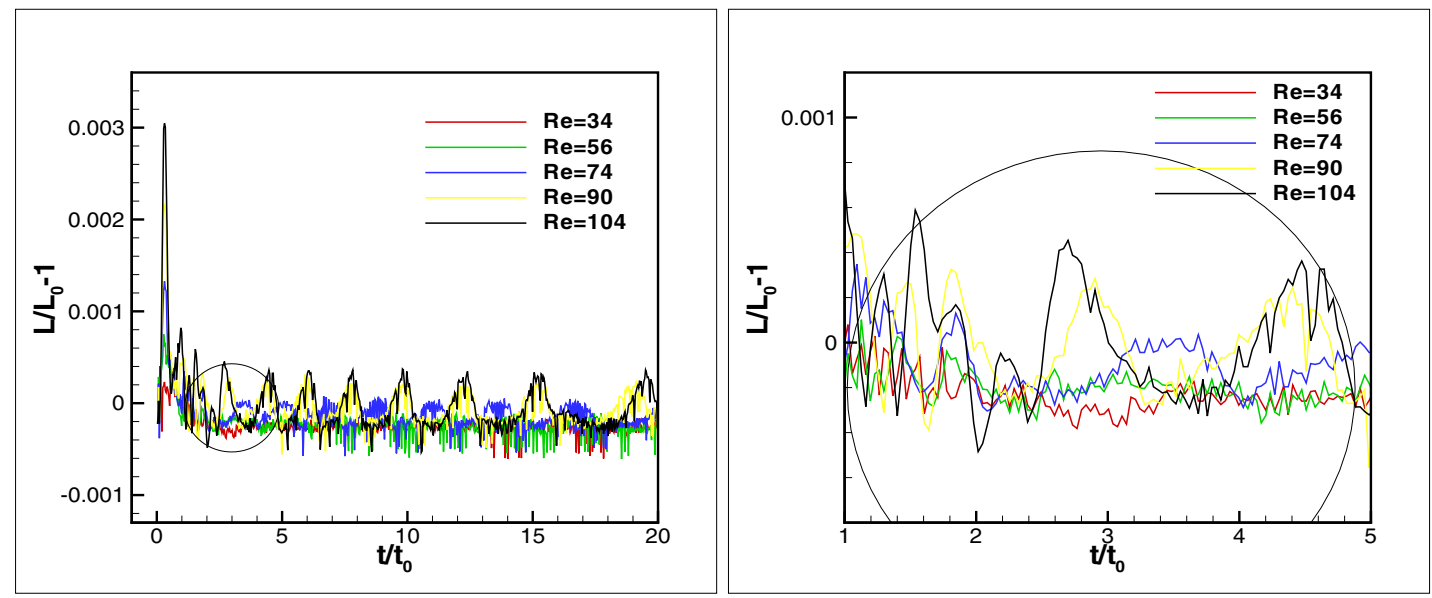

Fig. 8. Comparison of the fiber curl index as a function of time among different Reynold numbers.

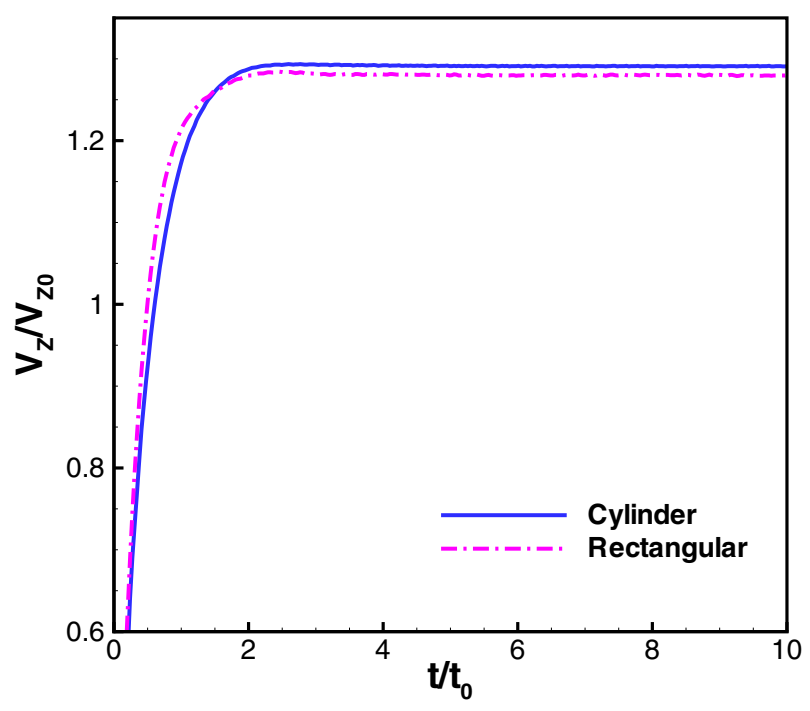

Fig. 9. Comparison of the settling velocity as a function of time between the rectangular and cylindrical fibers. The two fibers have the same length and cross section area (same volume) but with different shapes.

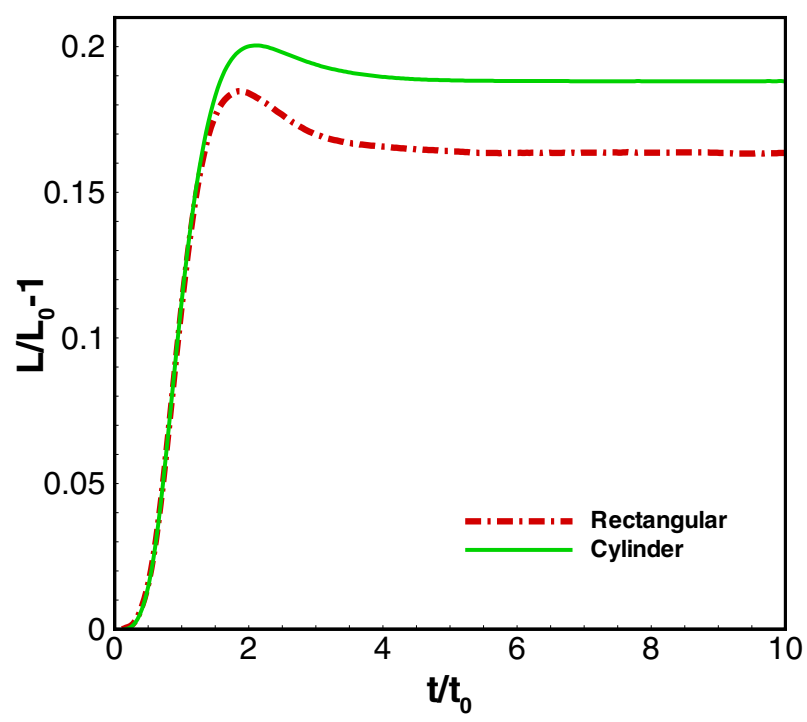

Fig. 10. Comparison of the curl index between the rectangular and cylindrical fibers. The two fibers have the same length and cross section area (same volume) but with different shapes.

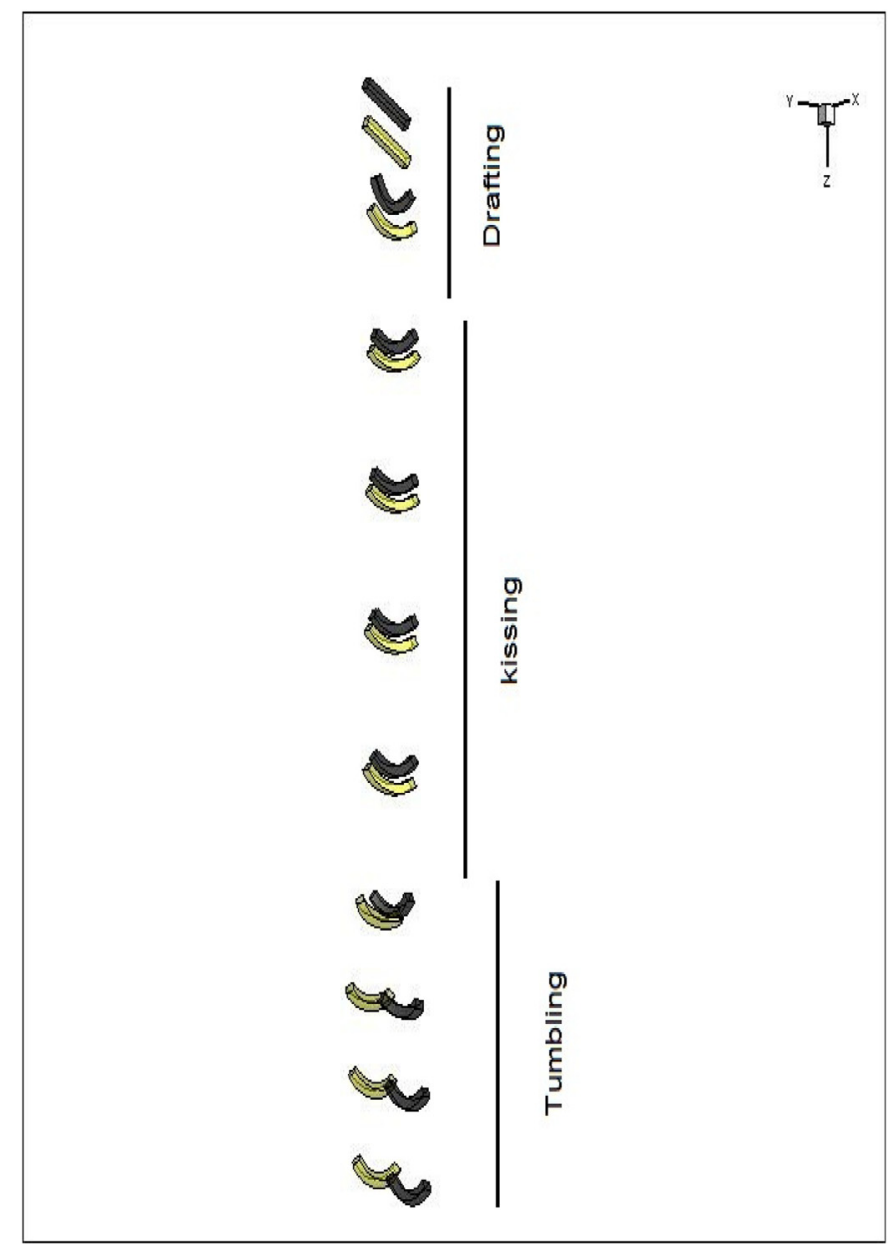

Fig. 11. The DKT scenarios are shown at the different time instants for the two fibers with $E I=0.029$.

where $l$ is the coordinate variable along the fiber length direction; $w$ is the displacement, due to bending, perpendicular to the fiber length and width directions; $F_{f}$ is the non-dimensional hydrodynamic force distributed on the fiber per unit length; $D$ is the thickness of the fiber; $B$ is its width; the normalized $E I=E I_{l} /\left(\rho_{f} V_{0}^{2} L H\right)$; $H$ is the volume of the fiber; $E I_{l}$ is the rigidity; $F r=V_{0}^{2} /(L g)$ is the Froude number, which is the ratio of the fluid inertia to the gravity; $g$ is the gravity acceleration. It is seen that the first term of the 

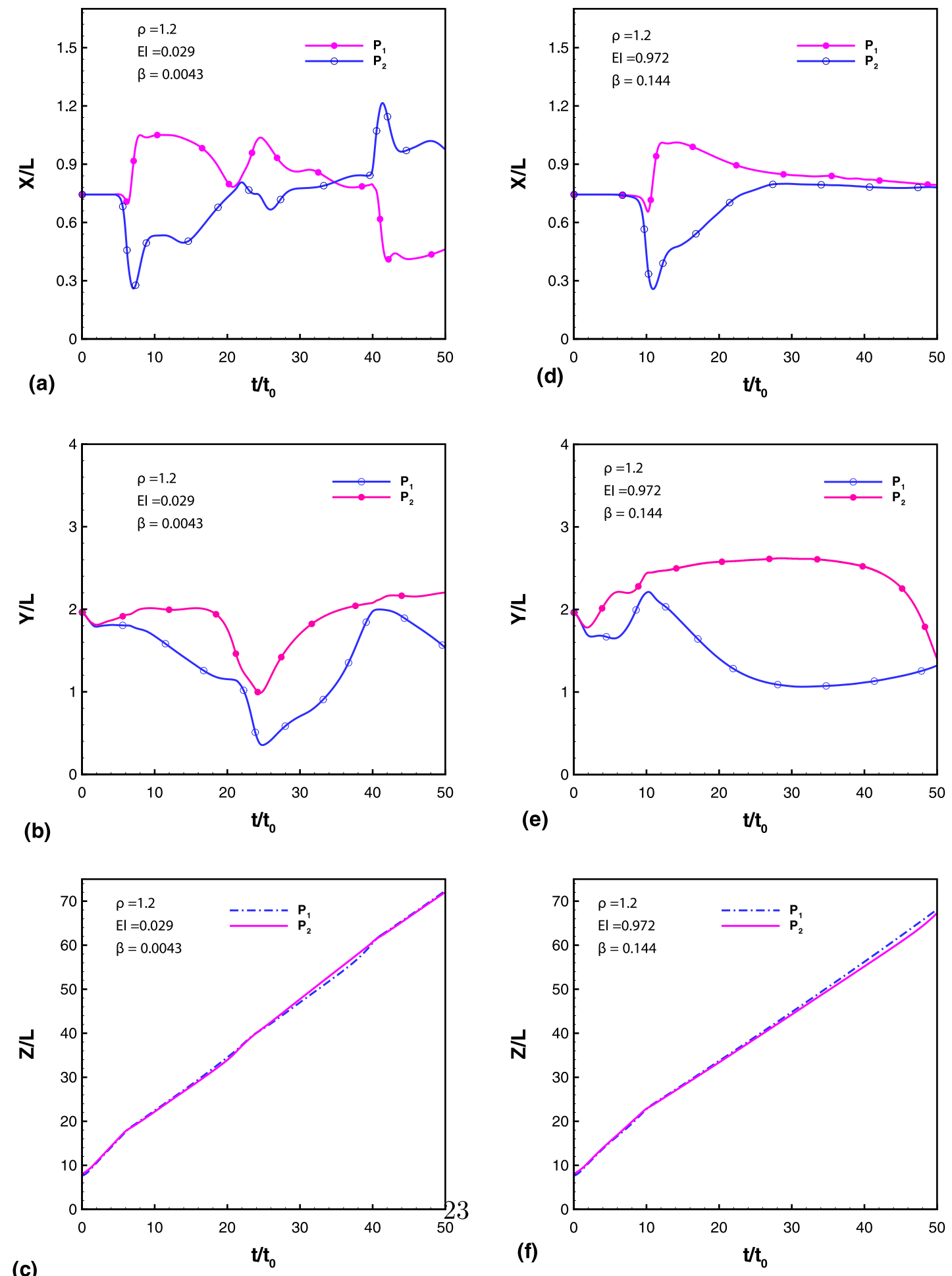

Fig. 12. Comparison of the position $(\mathrm{X}, \mathrm{Y}, \mathrm{Z})$ of the mass centers of two fibers as a function of time between two cases of $E I=0.029$ and $E I=0.972$.

left side of Eq. (3) is related to fiber rigidity and the second term is a fiber inertial term while the first term of the right side is associated with the hydrodynamic force and the second term comes from the gravity or buoyancy force. The fiber deformation and motion can be determined by a normalized rigidity EI, the fiber and fluid inertia, hydrodynamic force, and the gravity force. The gravity force drives fibers to settle down vertically and the fibers may be deformed due to their elasticity while the fiber rigidity and fluid forces may resist the deformation.
A non-dimensional elasto-gravitation number $\beta$ is defined by a ratio of the rigidity $E I$ to the buoyancy force as

$\beta=\frac{E I}{\left(\rho_{s} / \rho_{f}-1\right) / F r}$.

A larger elasto-gravitation number may result in a smaller deformation and vice verse. This number is useful for analysis of effects of the rigidity and gravity on the deformation. 

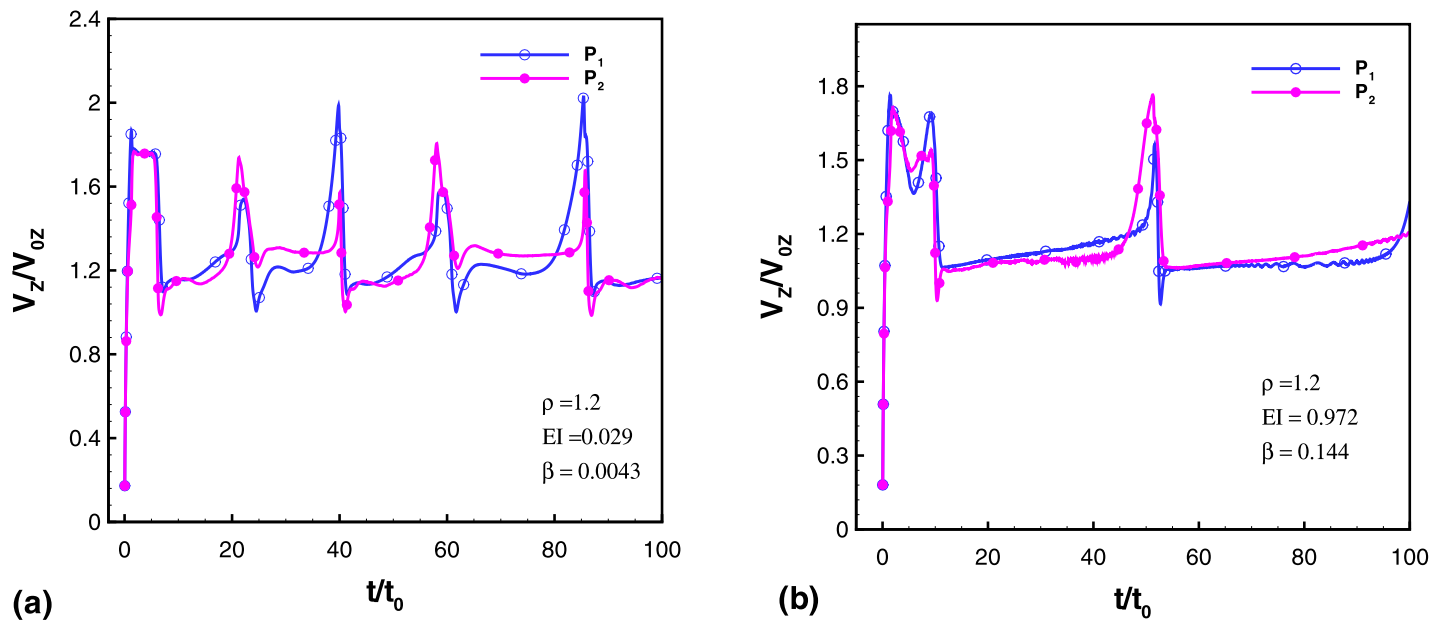

Fig. 13. Comparison of the settling velocities of two fibers as a function of time between the two cases of a) $E I=0.029$ and $b$ ) $E I=0.972$.

\subsection{Lattice-Boltzmann lattice-spring model}

The Navier-Stokes equations describe the motion of viscous fluids. The equations could be solved by many computational fluid dynamics methods [11]. In particular, the lattice Boltzmann (LB) method has been proven to be especially effective for solving the fluid flows interacting with complex solid boundaries when the March number is smaller than 0.3 [12-21,21,22].

A group of lattice nodes is used to represent fluid. Each node has distribution functions $f_{\sigma}$ and discrete micro-velocity $\mathbf{e}_{\sigma}$, where $\sigma$ depends on the chosen lattice model. The Boltzmann equation with BGK [23] single relaxation time model is

$$
\begin{aligned}
f_{\sigma}\left(\mathbf{r}^{l}+\mathbf{e}_{\sigma}, t+\delta t\right)= & f_{\sigma}\left(\mathbf{r}^{l}, t\right) \\
& -\frac{1}{\tau}\left[f_{\sigma}\left(\mathbf{r}^{l}, t\right)-f_{\sigma}^{e q}\left(\mathbf{r}^{l}, t\right)\right]+\delta t \mathbf{F}_{\sigma}\left(\mathbf{r}^{l}, t\right),
\end{aligned}
$$

where $\tau$ is the relaxation time; $\mathbf{F}_{\sigma}\left(\mathbf{r}^{l}, t\right)$ is a body force; $f_{\sigma}^{e q}\left(\mathbf{r}^{l}, t\right)$ is the equilibrium distribution function at node position $\mathbf{r}^{l}$ and time $t$ as

$f_{\sigma}^{e q}=\omega_{\sigma} \rho_{f}\left\{1+3\left(\mathbf{e}_{\sigma} \cdot \mathbf{u}\right)+\frac{9}{2}\left(\mathbf{e}_{\sigma} \cdot \mathbf{u}\right)^{2}-\frac{3}{2}(\mathbf{u} \cdot \mathbf{u})\right\}$,

where $\mathbf{u}$ is the fluid velocity. In this simulation, the D3Q15 lattice model is applied, the discrete velocities are given by

$\mathbf{e}_{\sigma}= \begin{cases}(0,0,0), & \sigma=0 \\ ( \pm 1,0,0),(0, \pm 1,0),(0,0, \pm 1), & \sigma=1 \\ ( \pm 1, \pm 1, \pm 1), & \sigma=2,\end{cases}$

and the weight coefficients are

$\omega_{\sigma}= \begin{cases}\frac{2}{9}, & \sigma=0 \\ \frac{1}{9}, & \sigma=1 \\ \frac{1}{72}, & \sigma=2 .\end{cases}$

According to Guo et al. [24],

$\mathbf{F}_{\sigma}=\left(1-\frac{1}{2 \tau}\right) \omega_{\sigma}\left(\frac{\mathbf{e}_{\sigma}-\mathbf{u}}{c_{s}^{2}}+\frac{\mathbf{e}_{\sigma} \cdot \mathbf{u}}{c_{s}^{4}} \mathbf{e}_{\sigma}\right) \cdot \mathbf{F}$,

where $\mathbf{F}$ is the body force and its component in the $\sigma$ direction is $\mathbf{F}_{\sigma} ; c_{s}^{2}=1 / 3$ where $c_{s}$ is the sound speed; the fluid density $\rho_{f}$ and momentum density $\rho_{f} \mathbf{u}$ are given by

$\rho_{f}=\sum_{\sigma} f_{\sigma}$,

$\rho_{f} \mathbf{u}=\sum_{\sigma} f_{\sigma} \mathbf{e}_{\sigma}+\frac{1}{2} \delta t \mathbf{F}$
The kinematic viscosity $v$ is related to the relaxation time $\tau$ and given by

$v=\frac{1}{3}\left(\tau-\frac{1}{2}\right) \delta t$

A flexible fiber is modeled by a lattice spring (LS) method. In the method, the fiber is discretized as individual particles with a mass of $m$. The particles are connected by bonding harmonic elastic springs and angular elastic springs to ensure that three body forces are added into the model so that it can handle the bending deformation [25]. The spring energy $U^{s}$ acted on the $i$ th node is given by

$U_{i}^{s}=\frac{1}{2} k_{s} \sum_{j}\left(\mathbf{r}_{i j}-\mathbf{r}_{0 i j}\right)^{2}$

where $k_{s}$ is the spring coefficient; $\mathbf{r}_{0 i j}$ is the equilibrium length of the spring between two neighboring particles $i$ and $j ; j$ is the neighboring solid particle of the $i$ th solid particle; $\mathbf{r}_{i j}=\mathbf{r}_{i}-\mathbf{r}_{j}$. The angular energy $U^{a}$ is given by

$U_{i}^{a}=\frac{1}{2} k_{a} \sum_{j} \sum_{k, k \neq j}\left(\theta_{i j k}-\theta_{0 i j k}\right)^{2}$

where $k_{a}$ is the angular bonding coefficient; $j$ and $k$ are the nearest neighboring solid particles of $i$ th solid particle; $\theta_{i j k}$ is the angle between the bonding vectors $\mathbf{r}_{i j}$ and $\mathbf{r}_{i k} ; \theta_{0 i j k}$ is the corresponding equilibrium angle. The elastic force $\mathbf{F}_{i}^{e}$ on the $i$ th solid particle can be computed from the gradient of the total energy by

$\mathbf{F}_{i}^{e}=-\nabla\left(U_{i}^{s}+U_{i}^{a}\right)$.

The elastic modulus of the solid body can be related to the spring and angular coefficients. The relationship between elastic modulus $E$ and shear modulus depends on the lattice structure. For a cubic lattice structure without diagonal bonds, we have

$E=\frac{k_{s}}{r_{0}}$

where $r_{0}$ is the constant unit length of spring lattice [25] if the solid material is isotropic.

In order to force the fluid velocity to be equal to the solid boundary velocity at the fluid-solid interface, a direct forcing term F should be added to the un-forced fluid velocity $\mathbf{u}^{*}\left(\mathbf{r}^{b}, t\right)$ at the solid boundary position $\mathbf{r}^{b}$ and make the momentum summation equal to the solid boundary particle momentum $\mathbf{U}^{b}\left(\mathbf{r}^{b}, t\right)$ according to the Newton's second law. This is

$\rho_{f} \mathbf{u}\left(\mathbf{r}^{b}, t\right)=\rho_{f} \mathbf{u}^{*}\left(\mathbf{r}^{b}, t\right)+\delta t \mathbf{F}=\rho_{f} \mathbf{U}^{b}\left(\mathbf{r}^{b}, t\right)$, 


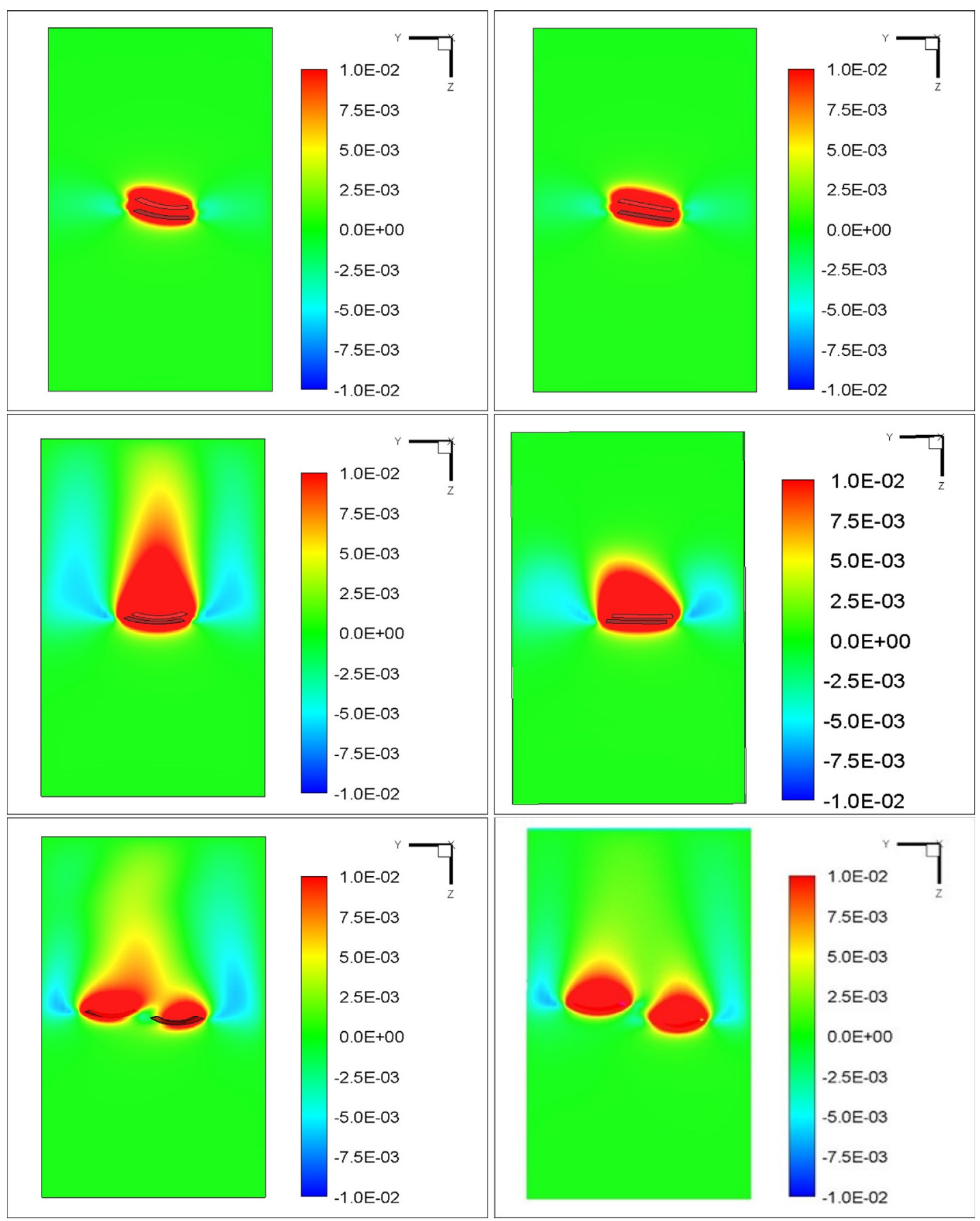

Fig. 14. Comparison of the contours of the vorticity in the X-direction on the YZ-plan between the two-fiber cases of $E I=0.029$ and 0.972 at three different time instants.

where $\mathbf{u}\left(\mathbf{r}^{b}, t\right)$ is the forced fluid velocity at $\mathbf{r}^{b}$. The un-forced velocity is calculated by

$\rho_{f} \mathbf{u}^{*}=\sum_{\sigma} f_{\sigma} \mathbf{e}_{\sigma}$

Therefore, the direct force on fluid can be calculated by

$\mathbf{F}\left(\mathbf{r}^{b}, t\right)=\frac{\rho_{f}\left(\mathbf{U}^{b}\left(\mathbf{r}^{b}, t\right)-\mathbf{u}^{*}\left(\mathbf{r}^{b}, t\right)\right)}{\delta t}$.

The force acting on the solid boundary node by fluid is $-\mathbf{F}\left(\mathbf{r}^{b}, t\right)$. The un-forced fluid velocity $\mathbf{u}^{*}$ at the position of the solid boundary node $\mathbf{r}^{b}$ is presented by

$\mathbf{u}^{*}\left(\mathbf{r}^{b}, t\right)=\int_{\Pi} \mathbf{u}^{*}\left(\mathbf{r}^{l}, t\right) D\left(\mathbf{r}^{l}-\mathbf{r}^{b}\right) d \mathbf{r}^{l}$, where $\mathbf{r}^{l}$ is a variable.

Since the fluid node position may not be coincided with the solid particle position, the fluid velocity on the boundary solid node can be approximately interpolated from the fluid velocities of the surrounding solid boundary nodes by using a discrete Dirac Delta function [26]

$D(\mathbf{r})= \begin{cases}\frac{1}{64 h^{3}}\left(1+\cos \frac{\pi x}{2 h}\right)\left(1+\cos \frac{\pi y}{2 h}\right)\left(1+\cos \frac{\pi z}{2 h}\right) & |\mathbf{r}| \leq 2 h \\ 0 & \text { otherwise }\end{cases}$

where $h$ is the one unit length of fluid lattice. The fluid nodes are within a spherical volume $\Pi$ with a radius of $2 h$, centered at a given solid node $\mathbf{r}^{b}$. After the fluid forcing term is calculated by 


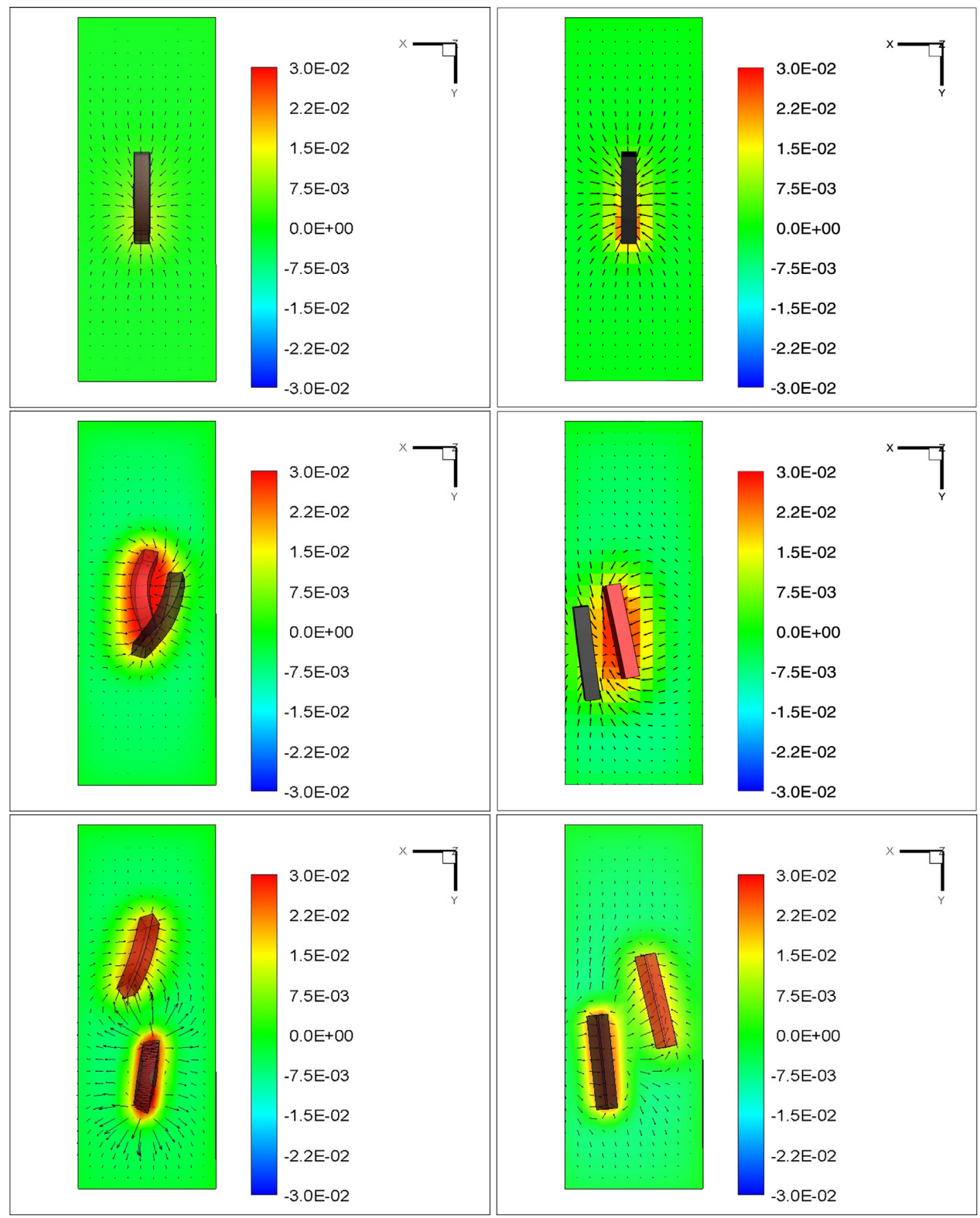

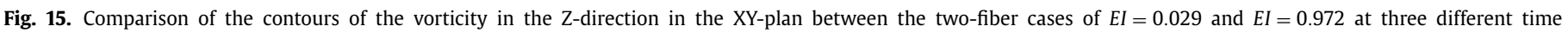
instants.

using Eq. (19) and the fluid velocity is computed by using Eq. (11), the forcing term components $\delta t \mathbf{F}_{\sigma}$ are added into fluid following Eq. (5). Next, the discrete Dirac delta function is utilized again to distribute the reaction force to the surrounding fluid nodes

$\mathbf{F}\left(\mathbf{r}^{l}, t\right)=\int_{\Gamma} \mathbf{F}\left(\mathbf{r}^{b}, t\right) D\left(\mathbf{r}^{l}-\mathbf{r}^{b}\right) d \mathbf{r}^{b}$,

where $\mathbf{F}\left(\mathbf{r}^{l}, t\right)$ is the distributed force and $\Gamma$ is a sphere with a radius of $2 h$, located at $\mathbf{r}^{l}$.

In addition, to avoid two fibers overlap during simulations, the repulsive portion of the Lennard-Jones potential is employed be- tween the solid particles in different fibers.

$U_{L J}=\epsilon\left(\frac{\Sigma}{\mathbf{r}_{i j}}\right)^{12}$,

where $\Sigma$ is a force interaction diameter and $\epsilon$ is the parameter of the intensity of the repulsion. The total force on a discretized solid particle $i$ is

$\mathbf{F}_{i}^{T}=-\mathbf{F}_{i}\left(\mathbf{r}^{b}\right)+\mathbf{F}_{i}^{e}+\mathbf{F}_{i L J}$,

where $\mathbf{F}_{i L J}$ is the force on the $i$ th particle due to the repulsive Lennard-Jones potential. Last, a leap frog algorithm is taken to update the position and velocity of each solid particle at each time 
Table 1

Variation of fiber and simulation box sizes for resolution tests.

\begin{tabular}{llll}
\hline Fiber size $(B, D, L)$ & Simulation box size $\left(N_{x} \cdot N_{y}, N_{z}\right)$ & Time step unit $(\mathrm{s})$ & Length unit $(\mathrm{cm})$ \\
\hline$(7,7,41)$ & $(60,160,650)$ & 0.000130 & 0.00806 \\
$(5,5,29)$ & $(43,114,464)$ & 0.000182 & 0.0113 \\
$(4,4,23)$ & $(34,91,371)$ & 0.000229 & 0.0141 \\
\hline
\end{tabular}

Table 2

Variation of three different rigidity.

\begin{tabular}{lll}
\hline EI & Elasto-gravitation number $(\beta)$ & Terminal velocity \\
\hline 0.048 & 0.0036 & 0.0575 \\
0.50 & 0.0072 & 0.056 \\
1.166 & 0.086 & 0.055 \\
\hline
\end{tabular}

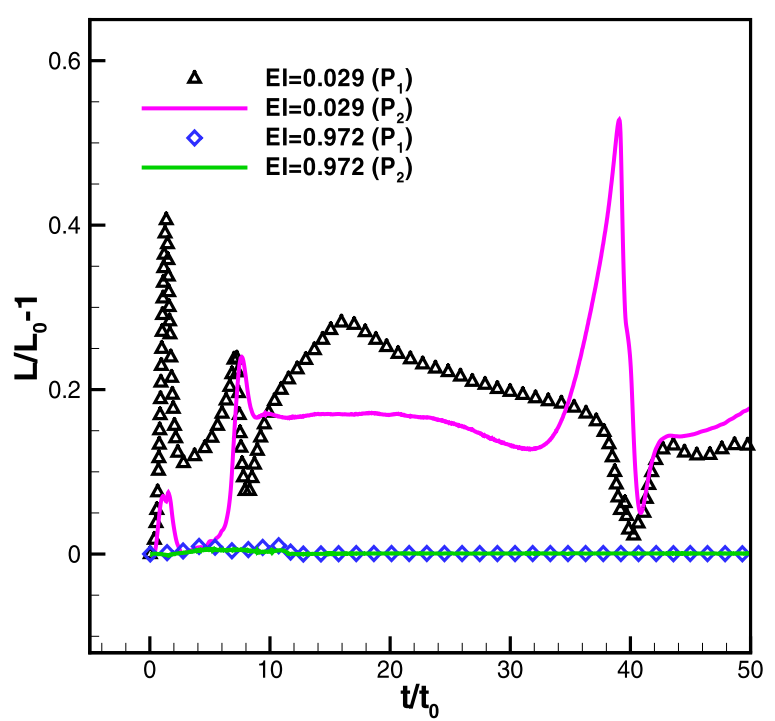

Fig. 16. A comparison of the curl indexes as a function of time between the two cases of $E I=0.029$ and 0.972 .

step. The details about the leap frog algorithm is referred to Chapter 3 of the book by Allen and Tildesley [27].

\section{Simulation}

The LBLS method has been extensively validated by comparing simulation results with existing theoretical and experimental results in our previous publications [28-30]. The Forgacs and Masons experimental results[31,32] such as springy, snake $C$ and $\mathrm{S}$ shapes, coiled rotation without entanglement, helix rotation projects, coiled rotation with entanglement in a shear flow were recovered in our simulations [30]. Our simulation results were well compared with those from a flexible particle method [33], with Jeffery's theory of fiber rotation in a shear flow [34], and with fiber flapping theory of Wiggins, Riveline, and Goldstein [35]. With a great confidence, the method is employed again for the present work.
Table 4

Variation of rigidity of a two-fiber system.

\begin{tabular}{llll}
\hline Density & Rigidity & Elasto-gravitation numbers & Settling velocity \\
1.2 & 0.029 & 0.0043 & 0.038 \\
1.2 & 0.972 & 0.144 & 0.032 \\
\hline
\end{tabular}

\subsection{Simulation setup}

An infinitely long rectangular simulation box of $\left(N_{x}, N_{y}, N_{z}\right)=$ $(60,160,650)$ is used. a fiber with zero velocity is initial located at the center of the simulation box, then released, and settles down in the vertical or Z-direction by the gravity. Whenever the fiber moves down one grid distance, the fluid layer at the top grids is removed and another fluid layer is added in the bottom grids so that the distance between the fiber mass center and the top/bottom of the simulation box is always kept at $7.9 \mathrm{~L}$ during the simulation. Meanwhile, to ensure the free surface for the top fluid flow, the gradient of the fluid velocity in the vertical direction is satisfied by $\frac{\partial V_{z}}{\partial z}=0$ at the top fluid layer. The bottom fluid velocity is set as zero. Two walls are set at the ends of the simulation box in the $\mathrm{X}$-direction and periodic boundary conditions are imposed in the Y-direction.

To select appropriate grids, three different sizes of the fiber and simulation box are used to test simulation resolution for the same case of $E I=0.048, \beta=0.0036, \rho=\rho_{s} / \rho_{f}=1.4$, and $R e=58.94$. The sizes of the fibers are $(B, D, L)=(7,7,41),(5,5,29)$, and $(4,4$, 23 ) and their corresponding simulation box sizes are $\left(N_{x}, N_{y}, N_{Z}\right)=$ $(8.57 B, 22.86 B, 15.85 \mathrm{~L})$ as shown in Table 1 . The simulation length unit is 1.75 times and the time step unit is 1.76 times smaller for the fine grid case than for the coarse grid case. The results of the sedimentation velocity of the mass center as a function of time are compared in Fig. 1 among the fine, middle, and coarse grid cases. No significant difference is observed, indicating the resolution is enough even using coarse grids. However, to guarantee accuracy, the fine grids of the fiber size of $(B, D, L)=(7,7,41)$ and its corresponding simulation box size of $(60,160,650)$ is used in this work.

\subsection{Single fiber}

\subsubsection{Effects of flexibility}

To investigate the effect of flexibility on sedimentation of a single fiber, the simulations are conducted in the same conditions except that the rigidity is varied at three different levels, collected in Table 2.

In these cases, three levels of the rigidity $E I=0.048,0.50$, and 1.166 are used, respectively. The aspect ratio of the fiber is kept at $\kappa=L / D=5.857$ and the density ratio of the solid to fluid is fixed

Table 3

Variation of fiber density or Reynolds numbers.

\begin{tabular}{llll}
\hline Reynolds number & Fiber density $\rho=\rho_{s} / \rho_{f}$ & Elasto-gravitation nembers & Settling velocity \\
\hline 34 & 1.1 & 0.24 & 0.0216 \\
56 & 1.2 & 0.072 & 0.055 \\
74 & 1.3 & 0.048 & 0.073 \\
90 & 1.4 & 0.036 & 0.088 \\
104 & 1.6 & 0.029 & 0.102 \\
\hline
\end{tabular}



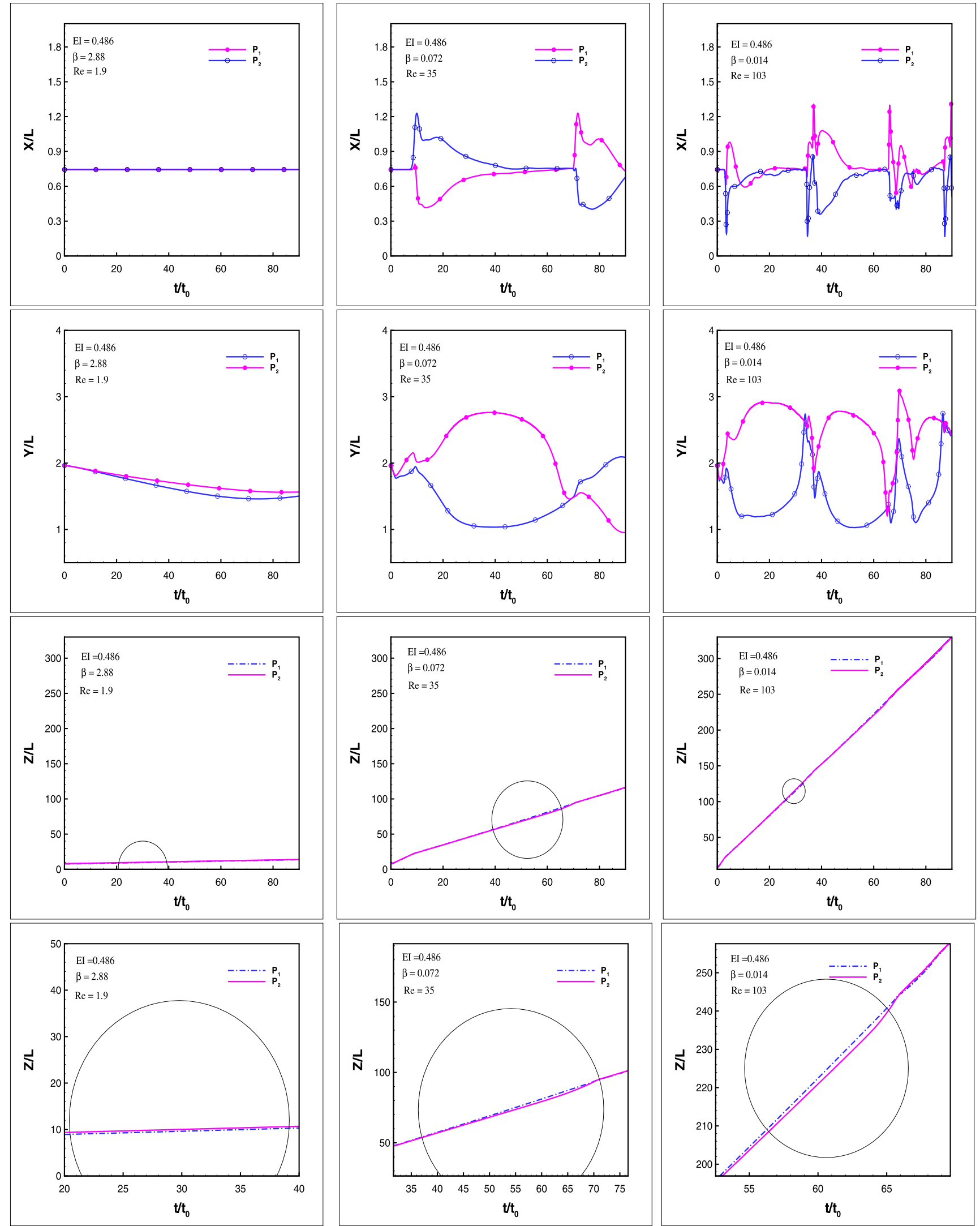

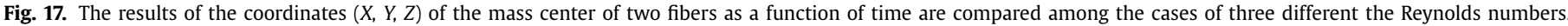



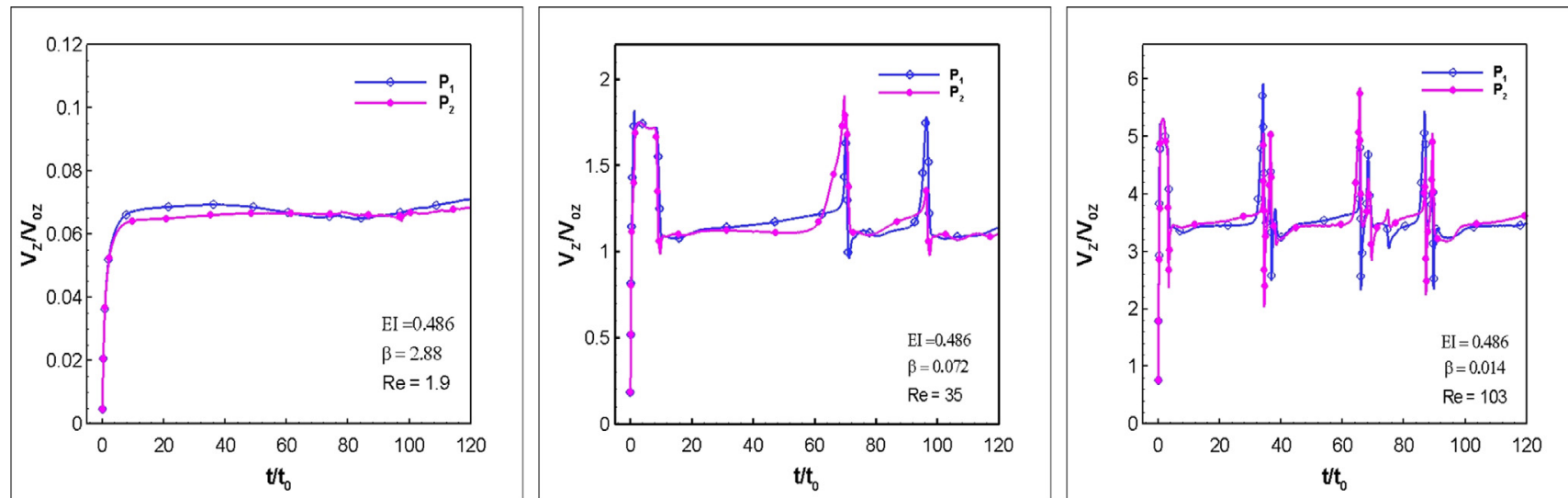

Fig. 18. A comparison of settling velocity of two fibers among cases of $R e=1.9,35$, and 103 where the rigidity is fixed at $E I=0.486$.
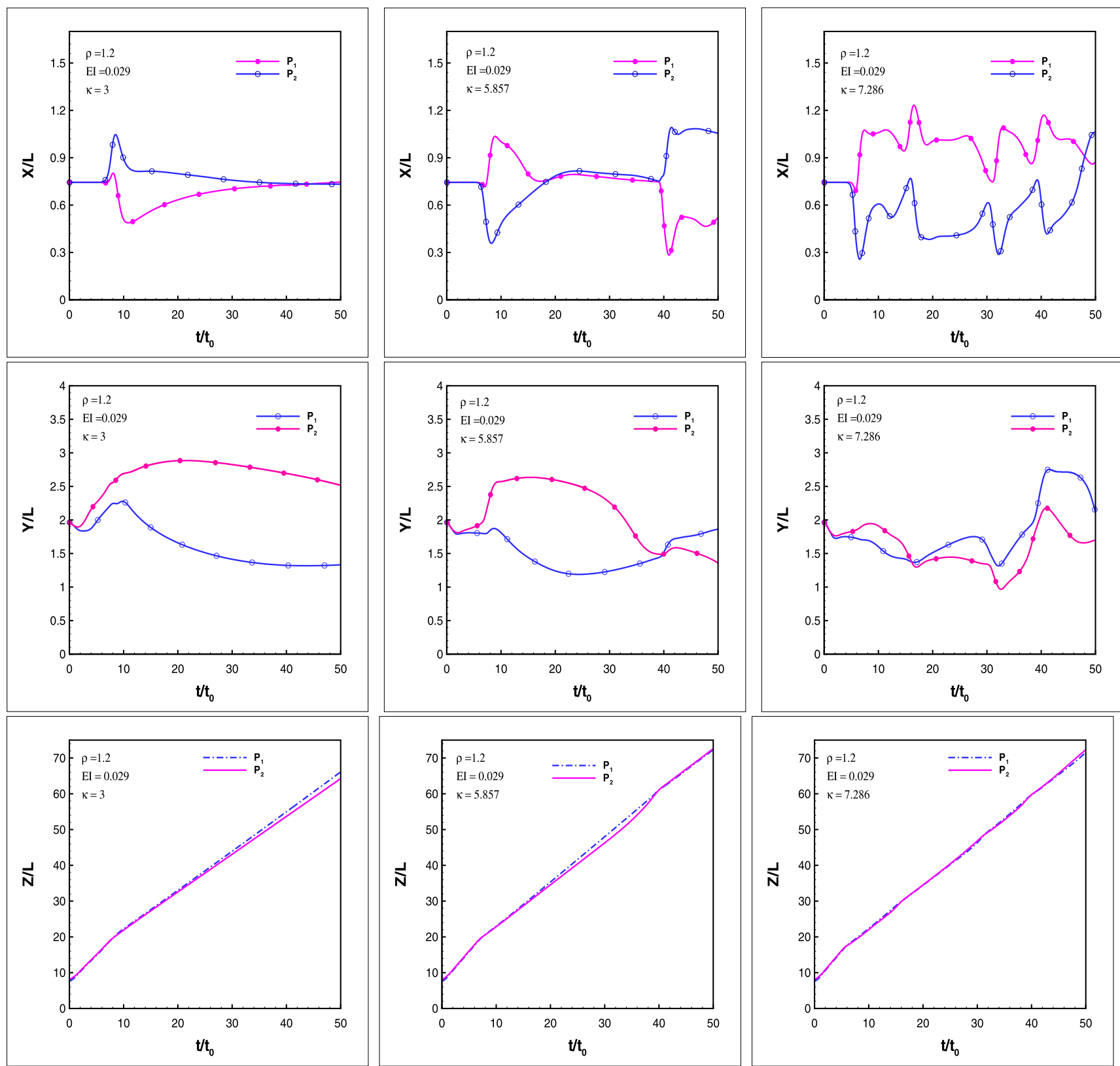

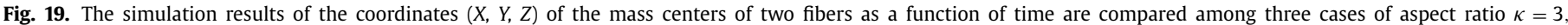
5.857, and 7.286. The rigidity is fixed at $E I=0.029$. 

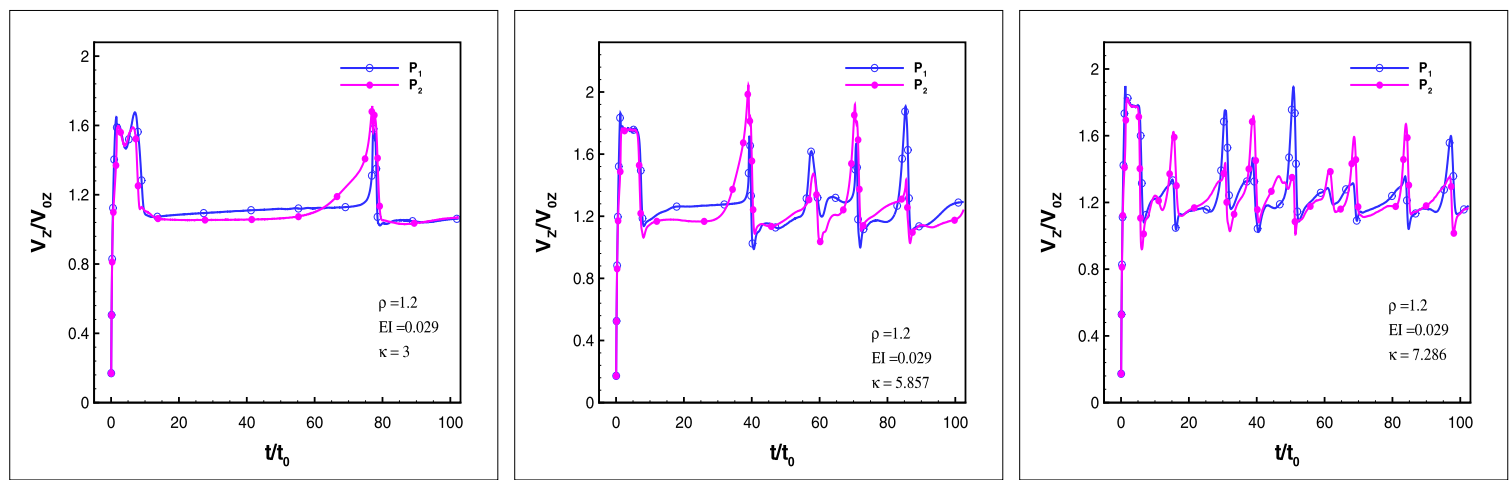

Fig. 20. A comparison of the settling velocity as a function of time among the three cases with different fiber aspect ratios.

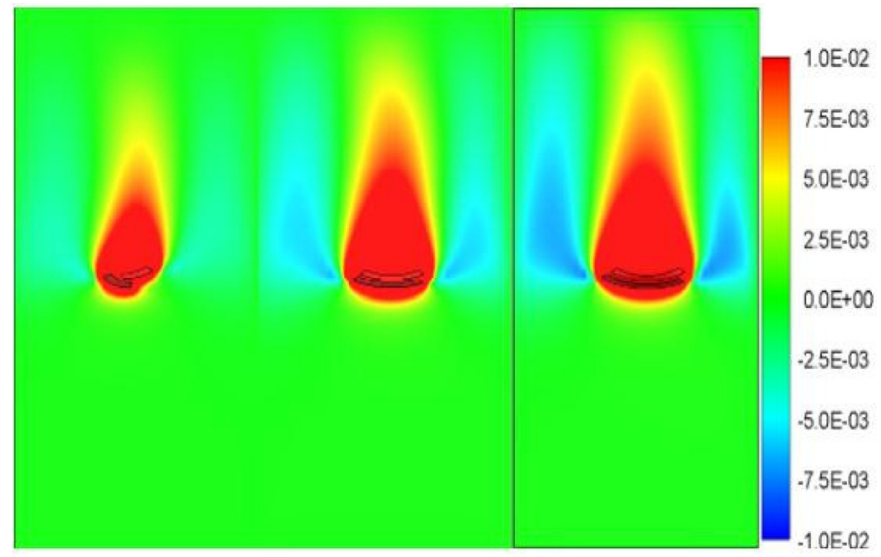

Fig. 21. A comparison of the contours of the vorticity in the X-direction among the three cases with different fiber aspect ratio.

at $\rho=\rho_{s} / \rho_{f}=1.4$. The results of the settling velocity of the mass center of the fiber as a function of time are plotted and compared in Fig. 3. In the figure, the settling velocity is normalized by the settling velocity $V_{0 z}$ in a Stokes flow. The Stokes velocity is obtained by a simulation where the second order term of the velocity in Eq. (6) and (9) are set as zero. The time is normalized by $t_{0}=L / V_{0 z}$.

When a fiber settles, it pushes the fluid in the front area and creates a higher pressure in the front of the fiber and a lower pressure in the back of the fiber. The pressure difference will try to move the fluid from the high pressure area to the low pressure
Table 5

Variation of fiber density for a two-fiber system.

\begin{tabular}{lll}
\hline Fiber density & Elasto-gravitation numbers & Settling velocity \\
\hline 1.1 & 2.88 & 0.0019 \\
1.2 & 0.072 & 0.034 \\
1.4 & 0.014 & 0.1 \\
\hline
\end{tabular}

area. However, the fluid in the fiber center is not easy to flow to the back side due to the resistant of the solid body. Only the fluid around the fiber tip area can flow easily to the back side of the fiber and form a circular back flow so that tip vortices are formed as show in Fig. 2. The contours of vortices are compared among the three different levels of rigidity in Fig. 4. It seems that the most flexible fiber has the largest vorticity.

A flexible body can be deformed more easily and fitted more conformably with the streamlines than a rigid fiber. Therefore, the settling velocity increases as the fiber flexibility increases as shown in Fig. 3. It is noted that the flexibility can be defined by $F_{b}=L / E I_{l}$.

Fig. 5 shows the results of curl index $C$ as a function of time. The curl index is defined by $C=L / L_{0}-1$, where $L_{0}$ is the end-toend distance of the curved fiber. The results clearly show that the fiber with $E I=0.048$ has largest deformation and is the most flexible fiber while the fiber with $E I=1.166$ has the little deformation and is the most rigid fiber.

\subsubsection{Effect of Reynolds number}

To study the effect of Reynolds number on sedimentation of single particle, five cases have been run in the same conditions except that the large Reynolds numbers are obtained by an increase
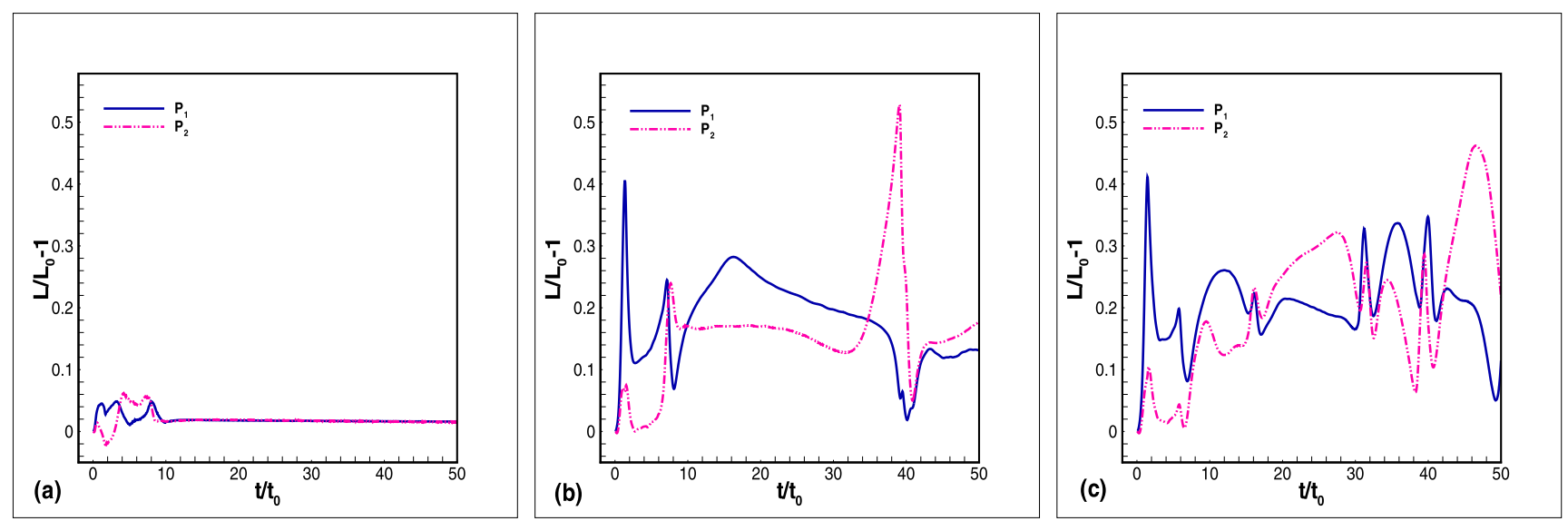

Fig. 22. A comparison of the curl index among different fiber aspect ratio a) $\kappa=3$, b) 5.857 , and c) 7.286. 

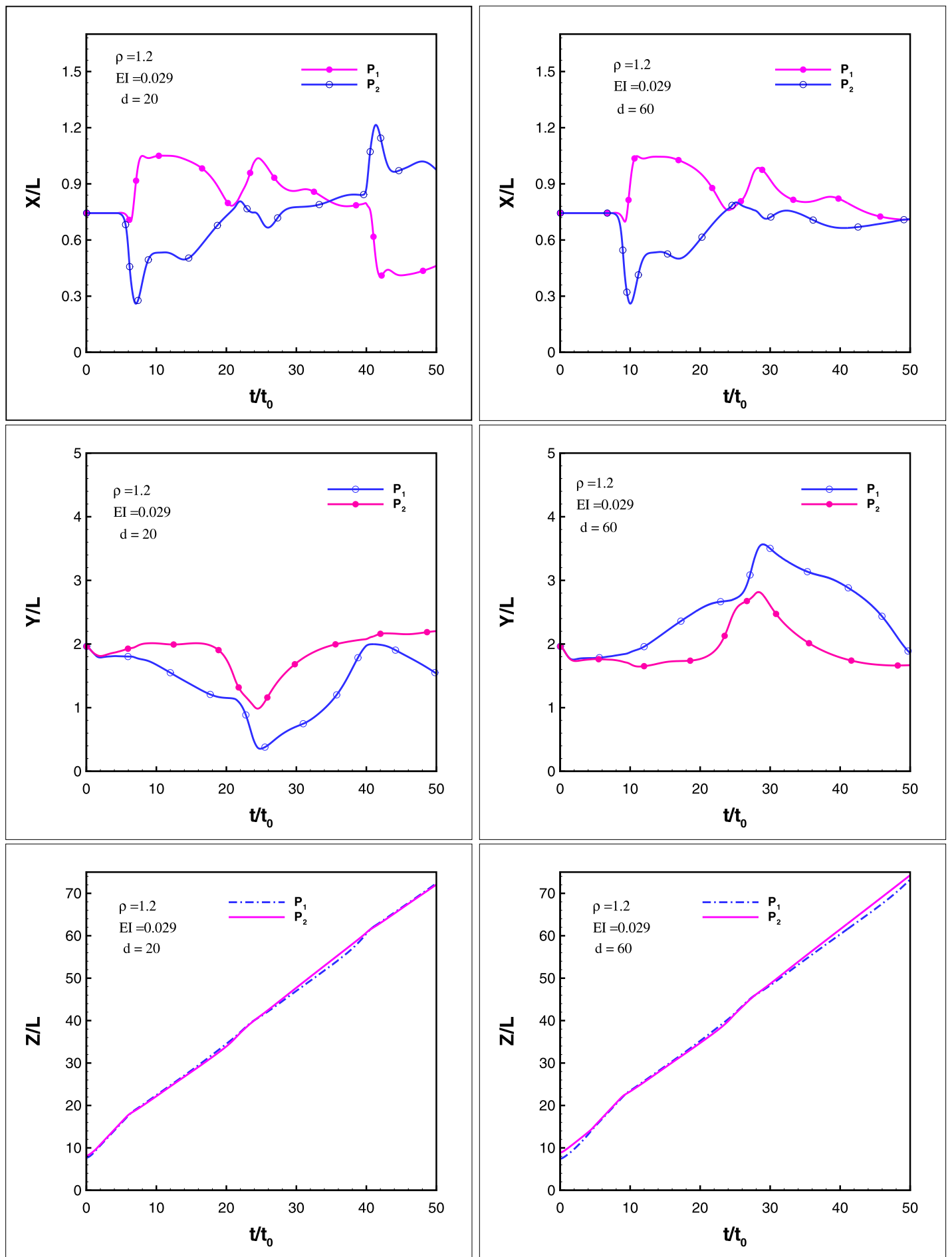

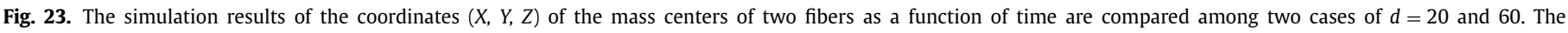
rigidity is fixed at $E I=0.029$.

in the density of the fiber. The elasto-gravitation numbers are calculated and shown in Table 3. The aspect ratio of the fiber is always fixed at $\kappa=5.58$.

The results of the sedimentation velocity as a function of time is displayed and compared in Fig. 6 among different Reynolds numbers. As expected, the settling velocity increases as the Reynolds number increases. The corresponding fluid structures are shown in Fig. 7. There are two vortices with opposite sign in the tip area behind the fiber. The intensity and size of the vortex pair due to back flows increase as the Reynolds number increases. The curl index $C$ increases as shown in Fig. 8, demonstrating that the deformation increases as the elasto-gravitation number decreases (see Table 3). In these cases, the gravitational force increases due to an increase in fiber density while the rigidity is fixed at $E I=0.971$.

\subsubsection{Effect of fiber shape}

To understand the effect of fibers shape on sedimentation, two different shape fibers are prepared. One is cylindrical and the other is rectangular. They have the same length $L=41$ and the same 

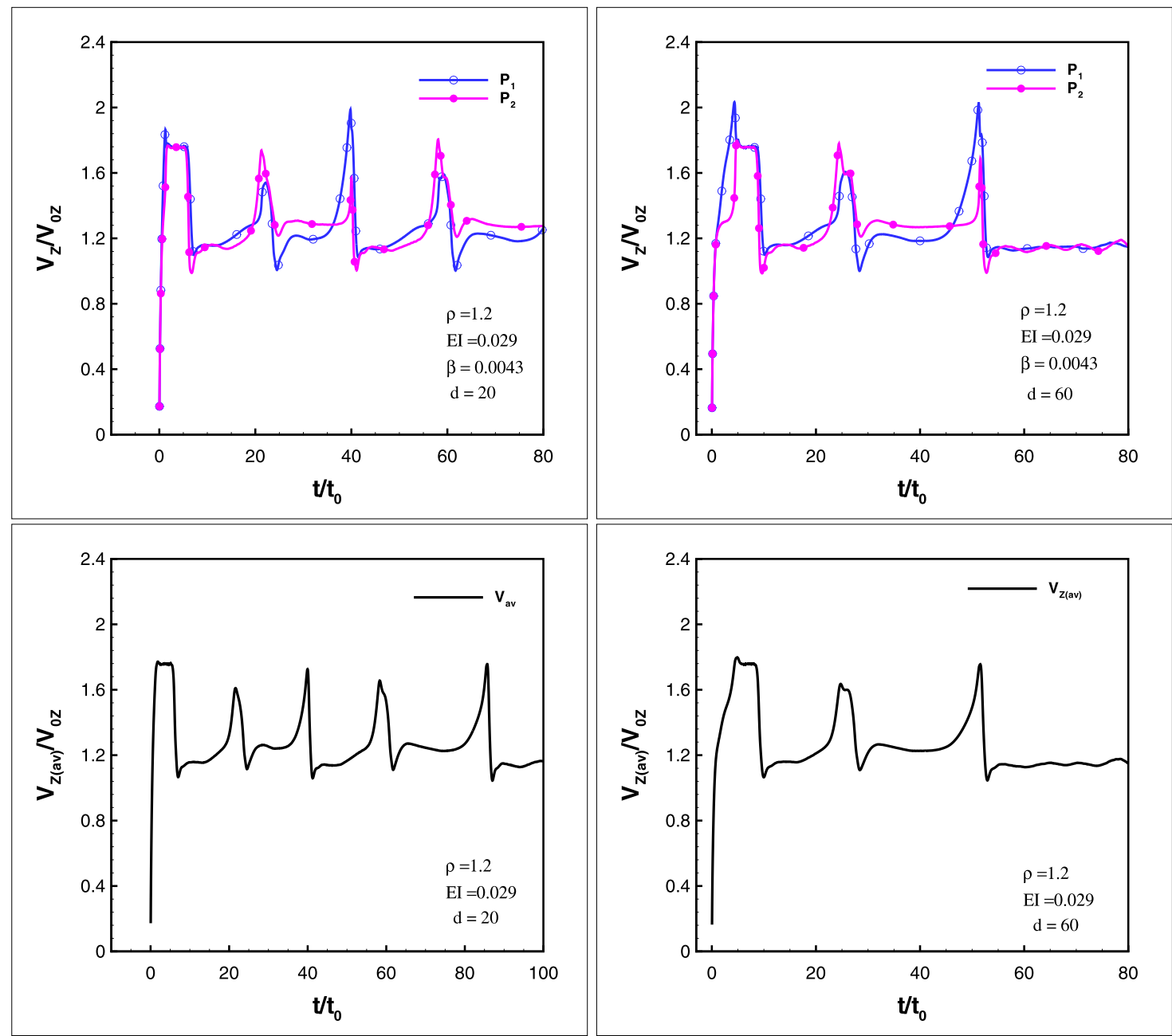

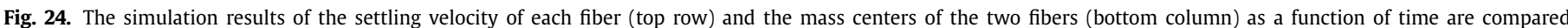
between the cases with the two different initial distances $d=20$ and 60 .
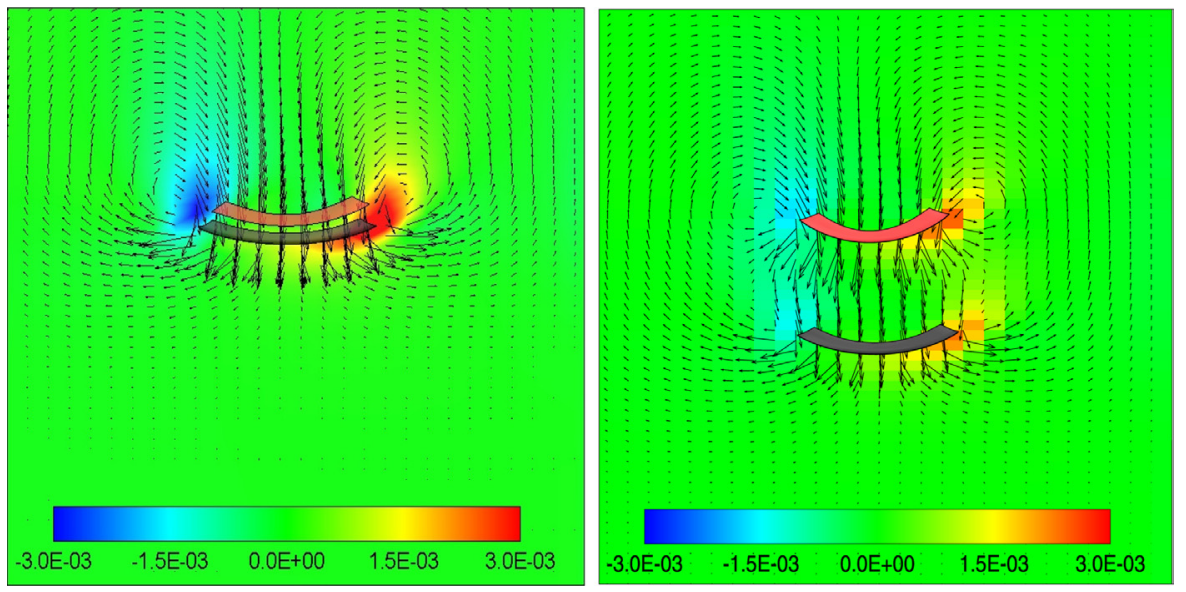

Fig. 25. A comparison of the contours of vorticity of two fibers settling between the two cases with different initial distances of a) $d=20$ b) 60 .

cross section area with different shapes. The simulations of the sedimentation of rectangular and cylindrical fibers, respectively, are conducted under the same conditions. The results as a function of time are plotted and compared in figure 9 for settling velocity and in Fig. 10. for the curl index It is shown that the cylindrical fiber settles slightly faster than the rectangular fiber since the elasto-gravitational number is $\beta=0.00423$ slightly smaller for the cylindrical fiber than $\beta=0.00432$ for the rectangular fiber. It is inferred that the elasto-gravitational number can be used to assess the deformation with different shapes. 


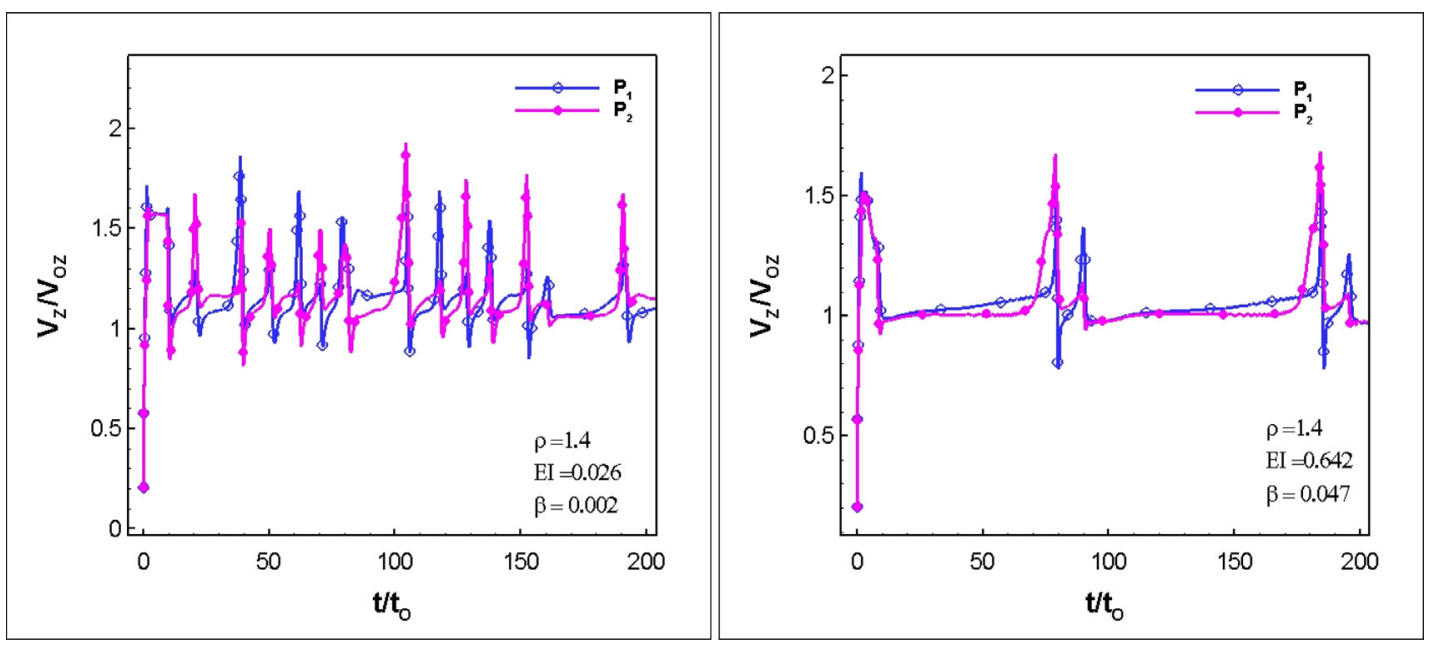

Fig. 26. A comparison of the settling velocity as a function of time among between the cases with different $E I=0.026$ and 0.642 while $\rho=1.4$.

Table 6

Variation of fiber aspect ratio.

\begin{tabular}{lllll}
\hline Fiber size & Aspect ratio & Elasto-gravitation numbers & Reynolds number & Settling velocity \\
\hline$(7,7,21)$ & 3.0 & 0.032 & 16.275 & 0.031 \\
$(7,7,41)$ & 5.857 & 0.0043 & 35.875 & 0.035 \\
$(7,7,51)$ & 7.286 & 0.0022 & 45.9 & 0.036 \\
\hline
\end{tabular}

Table 7

Effects of initial distance between two fibers.

\begin{tabular}{llll}
\hline Initial distance & Reynolds number & Elasto-gravitation number & Settling velocity \\
\hline 20 & 37.9 & 0.0043 & 0.038 \\
60 & 33.8 & 0.0043 & 0.033 \\
\hline
\end{tabular}

\subsection{Sedimentation of two fibers}

A series of simulations of dynamic motion of two fibers, either rectangular or cylindrical, are conducted in a three dimensional space by using lattice-Boltzmann lattice-spring method. The simulation boundary conditions are the same as the single fiber case. The two fibers with an initial separating distance settle down by the gravity at the middle of the simulation box. Both the distances between the top of the simulation box and the mass centers of the trailing fiber and between the bottom of the simulation box and the mass center of the leading fiber are kept at a constant of 7.9L during settling. The fiber flexibility, initial distance, Reynolds numbers, and fiber aspect ratios are varied and their effects on the dynamic behavior are investigated.

\subsubsection{Effects of flexibility on two fiber sedimentation.}

In order to study the effects of flexibility on sedimentation of two rectangular fibers, two simulations are conducted in the same conditions except that the rigidity is varied at two different levels of $E I=0.029$ and 0.97 (see Table 4). Each fiber has the same density $\rho=1.2$ and the same size of $(B, D, L)=(7,7,41)$. The aspect ratio of the fiber is fixed at $\kappa=5.857$. The two fibers are initially located in the center of the simulation box and the fiber axes are aligned along the horizontal direction initially. The initial distance between the two fibers is 20 lattices. Our results show that similar to spherical particles, after releasing, two fibers go through a DKT process as shown in Fig. 11 for the case of $E I=0.029$. This DKT phenomenon can take place repeatedly during the settling process.

To illustrate effects of rigidity on DKT scenarios, the results of position coordinates $(X, Y, Z)$ of the mass centers of two fibers as a function of time are plotted and compared between the two cases with different rigidity $E I=0.029$ and 0.972 in Fig. 12, and their corresponding settling velocities are compared in Fig. 13. It is clear that one crossing on the Z-curves, in Fig. $12 \mathrm{c}$ and $\mathrm{f}$, between two fibers $P_{1}$ and $P_{2}$ corresponds to a DKT process. There are more than one crossing on the Z-curves for both cases of $E I=0.029$ and 0.972 , signifying that the DKT scenarios takes place repeatedly during settling. Markedly, there are more numbers of crossings for the case of $E I=0.029$ than for the case of $E I=0.972$ in the same period of time, illustrating that that a stronger DKT interaction with fluid occurs for more flexible fibers. The coordinates of $\mathrm{X}$ and $\mathrm{Y}$ as a function of time are also plotted in the same figure, which shows that the distance between the two fibers decreases, then increases in cycles as the DKT scenario is repeated. The leading fiber with smaller value of the rigidity settles faster, creates a stronger wake, and cause a faster DKT due to a large suction effect of the wake, as compared with the more rigid fibers. The vertical velocity $V_{z}$ of the two flexible fibers as a function of time is plotted and compared in Fig. 13 between the two cases with different levels of rigidity. It shows that the fibers with smaller rigidity settle faster and have more numbers of the DKT, suggesting that the rigidity plays an important role in two fiber interactions with fluid during sedimentation.

The contours of vorticity in the X-direction on the YZ-plan at three different instants are shown and compared between the cases of $E I=0.029$ (left) and 0.972 (right) in Fig. 14 while the contours of the vorticity in the Z-direction on the XY-plane are shown in Fig. 15. The vorticity is larger for the case with smaller rigidity of $E I=0.029$ than for the case with a larger rigidity $E I=0.972$. The fluid structures are consistent with the fibers with smaller values of the rigidity having a stronger wake and faster DKT. 

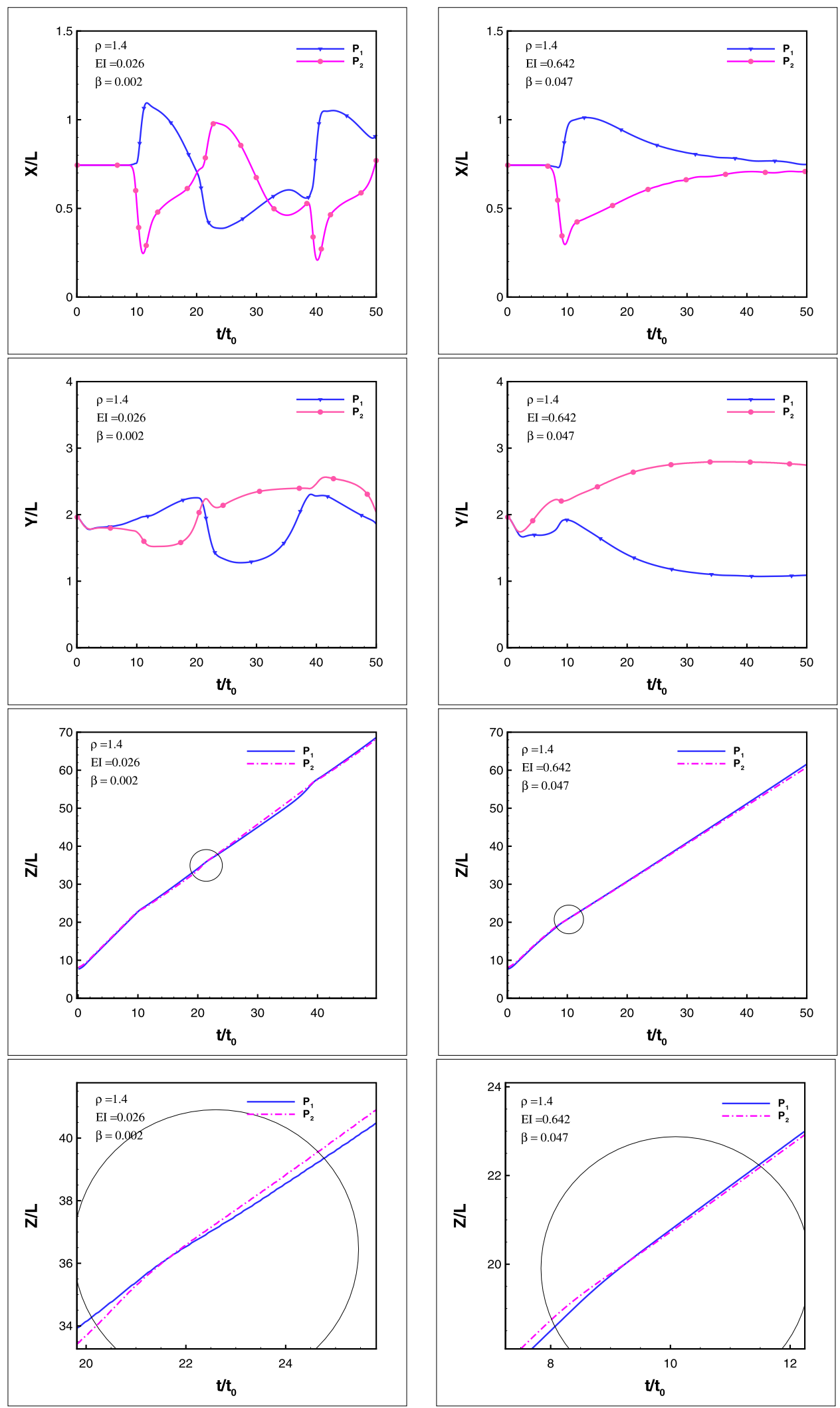

Fig. 27. A comparison of the position $(X, Y, Z)$ of the mass centers of two cylinder fibers as a function of time between two cases of $E I=0.026$ and $E I=0.642$. 
Table 8

variation of EI for two cylindrical fibers.

\begin{tabular}{lllll}
\hline Density & Reynolds number & Rigidity & Elasto-gravitation numbers & Settling velocity \\
\hline 1.4 & 53.3 & 0.026 & 0.002 & 0.052 \\
1.4 & 50 & 0.642 & 0.047 & 0.046 \\
\hline
\end{tabular}

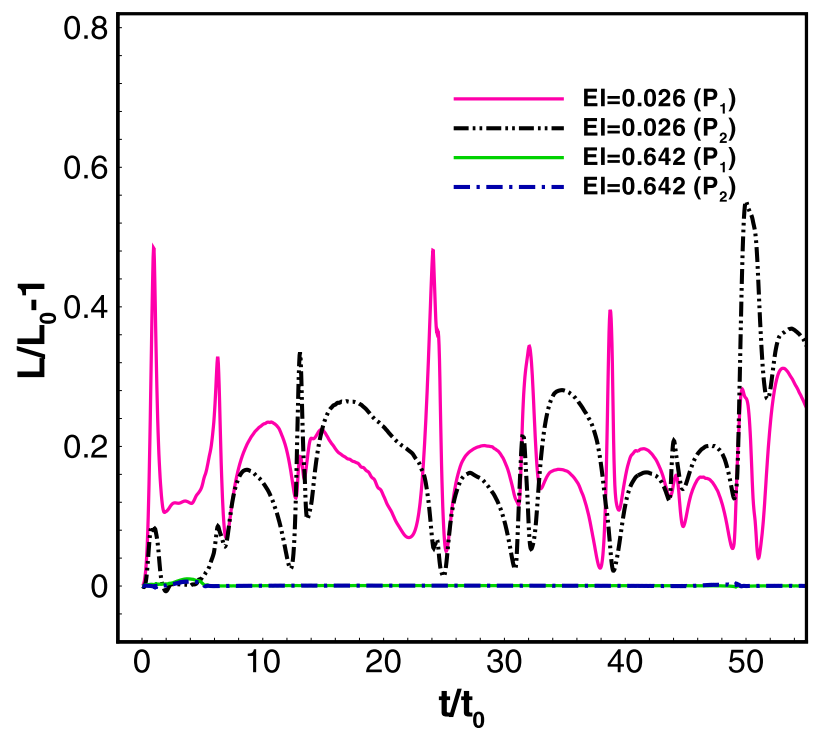

Fig. 28. A comparison of the curl indexes as a function of time between the two cases of $E I=0.026$ and $E I=0.642$.

The results of the curl index as functions of time at the two different levels of the rigidity are exhibited in Fig. 16. It is shown that the deformation is larger for the fibers with a smaller rigidity. As expected, deformation increases as the rigidity decreases.

\subsubsection{Effects of Reynolds numbers}

To assess the effects of the Reynolds number on the sedimentation of two fibers, additional simulations are conducted. Three cases are run in the same conditions and the fibers have the same rigidity of $E I=0.48$ except different Reynolds numbers. The large Reynolds numbers are obtained by an increase in the fiber density. The final Reynolds numbers are $R e=1.9,35$, and 103. The simulation parameters for the three cases are collected in Table 5.

The results of the coordinates $(X, Y, Z)$ of each fiber as a function of time are plotted and compared among the cases with three different Reynolds numbers $R e=1.9,35$, and 103 in Fig. 17.

The corresponding settling velocities as a function of time are plotted in Fig. 18.

These figures show that when the Reynolds number is small at $R e=1.9$, the DKT phenomenon occurs once only. As the Reynolds number increases, the number of the DKT increases. The tip vortices interacting with two fibers become stronger as the Reynolds number increases.

\subsubsection{Effects of fiber aspect ratio}

To assess the impact of aspect ratio on the dynamical behavior of fibers simulations are conducted at the same conditions except that the fiber length is varied at three different levels of $L=51,41$, and 21 so that the aspect ratio is varied at $\kappa=3.0,5.85$, and 7.28 (see Table 6).

The simulation results of coordinates $(X, Y, Z)$ as a function of time are compared among the three cases in Fig. 19 and the corresponding settling velocity is compared in Fig. 20. These figures show that all the cases go through the DKT process and the number of the DKT increases dramatically as the aspect ratio increases since the elasto-gravitational number decreases as the aspect ratio increases, resulting in a larger Reynolds number and stronger tip vortex interactions as shown in Fig. 21. The deformation also increases as the aspect ratio increases as shown in Fig. 22.

\subsubsection{Effects of initial distance}

To evaluate the effect of initial distance between two fibers on their motion, two simulations are conducted in the same rigidity $E I=0.029$ and the same conditions except that the initial distance is varied at $d=20$ and 60 lattice length (see Table 7).

The simulation results as a function of time are displayed and compared between the two cases in Fig. 23 for the coordinates $(X$, $Y, Z$ ) of the mass center of each fiber and in Fig. 24 for the settling velocity of each fiber.

It is shown that the settling velocity and number of the DKT decreases as the initial distance between the two fibers increases and that the Reynolds number in term of the velocity of the mass centers of two fibers decreases as the initial distance increases. It is understood that it takes a longer time for the trailing fiber to catch the leading fiber if the initial distance is larger. The larger initial distance may reduce the intensity of the tip vortex which has a suction effect on the trailing fiber, as shown in Fig. 25.

\subsection{Cylindrical fibers}

The LBLS method is also applied to the case of the sedimentation of two cylindrical fibers. The simulation parameters are collected in Table 8. The simulation results show that the dynamic behavior is similar to the rectangular fibers. For example, the comparison between the cases with $E I=0.026$ and 0.642 at fixed $\rho=$ 1.4 is shown in Fig. 26 for the settling velocity, in Fig. 27 for the coordinates $(X, Y, Z)$, and in Fig. 28 for the curl index. Again, the flexible fibers have more number of the DKT and have a larger settling velocity.

\section{Conclusion}

The sedimentation behavior of two flexible fibers in an infinitely long channel is simulated at moderate Reynolds numbers by using the LBLS method. In the method, the lattice Boltzmann method is employed to mimic the fluid behavior of Navier-Stokes equation and the lattice spring model is used to handle the deformation of flexible fibers, which are discretized as individual particles connected each other by harmonic bond and angular springs. The immersed boundary method is utilized to deal with the interfaces between the fluid and solid particles. In the simulations, the rigidity, fiber density, shape, initial vertical distance, and aspect ratio are varied at different levels and their effects on the DKT behavior are investigated. It is revealed that the DKT dominates the interaction between fluid and two flexible fibers and that the number of the DKT increases as the rigidity reduces, indicating a smaller rigidity induces stronger tip vortices, behind the fiber, which encourage a larger suction effect due to lower pressure and lead to a quicker DKT. It is also demonstrated that a larger aspect ratio with the same cross section area and shape results in more numbers of the DKT within the same time period and that the number of the DKT increases as the Reynolds number increases. In brief, the flexibility and gravity encourage a stronger tip vortices and a quick DKT. It is shown that the elasto-gravitational number 
can be used to consider the combinational effect of both rigidity and gravity on fiber deformation and motion.

\section{Acknowledgement}

Mr. Alhasan would like to express his appreciation to the Graduate Scholarship from the Iraq government. Mr. Luo and Wu would like to appreciate the 345 Graduate Assistantship from the Western Michigan University. Dr. Qi would like to acknowledge the support from HPC of the College of Engineering and Applied Science of the Western Michigan University. Dr. He would like to acknowledge the support from the Strategic Priority Research Program (Grant No. XDB22040104), Key Research Program of Frontier Sciences of the Chi-350 nese Academy of Sciences, National Natural Science Foundation of China (Nos. 11302238, 11232011).

\section{References}

11] Ayeni 00. Sedimentation of swarms of particles at low and moderate reynolds numbers. Louisiana State University: Agricultural and Mechanical College; 2013. Master's thesis.

[2] Rong X. Numerical study of flow in three-dimensional fiber networks and sedimentation of curved fiber by lattice boltzmann method. Western Michigan University; 2007. Master's thesis.

[3] Leal LG. Particle motions in a viscous fluid. Annu Rev Fluid Mech 1980;12:435

[4] Fortes AF, Joseph DD, Lundgren TS. Nonlinear mechanics of fluidization of beds of spherical particles. J Fluid Mech 1987;177(1):467-83.

[5] Feng YT, Han K, Owen DRJ. Coupled lattice boltzmann method and discrete element modelling of particle transport in turbulent fluid flows: computational issues. Int J Numer Methods Eng 2007;72(9):1111-34.

[6] Wang L, Guo Z, Mi J. Drafting, kissing and tumbling process of two particles with different sizes. Comput Fluids 2014;96:20-34.

[7] Koch D, Shaqfeh E. The instability of a dispersion of sedimenting spheroid. ] Fluid Mech 1989;209:521-42.

[8] Manikantan H, Li L, Spagnolie Se, Saintillan D. The instability of a sedimenting suspension of weakly flexible fibres. J Fluid Mech 2014;756:935-64.

[9] Switzer LH. Simulating systems of flexible fibers. The University of Wisconsin - Madison; 2002. Ph.D. thesis

[10] Wu T-H, Qi D. Advantages of a finite extensible nonlinear elastic potential in lattice boltzmann simulations. Hilltop Rev 2014;7(10)

[11] Oztekin D. The lattice boltzmann methods and their applications to fluid flows. Lehigh University; 2014. master thesis.

[12] He X, Luo L-S. A priori derivation of the lattice Boltzmann equation. Phys Rev E 1997:55:R6333(R).

[13] Koch DL, Ladd AJ. Moderate reynolds number flows through periodic and random arrays of aligned cylinders. J Fluid Mech 1997;349:31.

[14] Chen S, Doolen GD. Lattice boltzmann method for fluid flows. Annu Rev Fluid Mech 1998;30(1):329-64.
[15] Aidun CK, Clausen JR. Lattice-boltzmann method for complex flows. Annu Rev Fluid Mech 2010;42:439-72.

[16] Qi D, Liu Y, Shyy W, Aono H. Simulations of dynamics of plunge and pitch of a three-dimensional flexible wing in a low reynolds number flow. Phys Fluids 2010;22:091901.

[17] Qi D, He G, Liu Y. Lattice boltzmann simulations of sedimentation of a single fiber in a weak vertical shear flow. Phys Fluids, September 2013;25(9).

[18] Qi D. Lattice boltzmann simulations of particles in non-zero reynolds number flows. J Fluid Mech 1999;385:41.

19] Lallemand P, Luo L-S. Theory of the lattice boltzmann method: dispersion, dissipation, isotropy, galilean invariance, and stability. Phys Rev E 2000;61(6):6546

[20] Wu J, Aidun C. A numerical study of the effect of fibre stiffness on the rheology of sheared flexible fibre suspensions. J Fluid Mech 2010;662:123. 662, 123, 2010

[21] Wu J, Aidun CK. A method for direct simulation of flexible fiber suspensions using lattice boltzmann equation with external boundary force. Int J Multiphase Flow 2010;36(3):202-9. 2010;36:202-209.

[22] Wu T-H, Khani M, Sawalha L, Springstead J, Kapenga J, Qi D. A CUDA based implementation of a fluid-solid interaction solver: the immersed boundary Lattice-Boltzmann lattice-spring method. Commun Comput Phys 2018;23(4):980-1010. doi:10.4208/cicp.OA-2016-0251.

[23] Bhatnagar PL, Groos EP, Krook M. A model for collision processes in cases. i. small amplitude processes in charged and neutral one-component systems. Phys Rev 1954;94:511-25.

[24] Guo Z, Zheng C, Shi B. Discrete lattice effects on the forcing term in the lattice boltzmann method.. Phys Rev E 2002;65:046308.

[25] Wu TH, Guo RS, He GW, Liu YM, Oi D. Simulation of swimming of a flexible filament using the generalized lattice-spring lattice boltzmann method. J Theor Biol 2014;349:1-11.

26] Peskin CS. The immersed boundary method. Acta Numerica 2002:11:479-517.

[27] Allen MP, Tildesley DJ. Computer simulation of liquids. Oxford Science Publication; 1987.

[28] Luo Y, Wu T-H, Qi D. Lattice-Boltzmann lattice-spring simulations of flexibility and inertial effects on deformation and cruising reversal of self-propelled flexible swimming bodies. Comput Fliuds 2017;155(20):89-102.

[29] Wu T-H, Qi D. Lattice-Boltzmann lattice-spring simulations of influence of deformable blockages on blood fluids in an elastic vessel. Comput Fluids 2017;155(20):103-11.

[30] Tang Y, Wu T-H, He G, Qi D. Multi-flexible fiber flows: a direct-forcing immersed boundary-lattice Boltzmann lattice-spring approach. Multi-phase Fluids 2018;99:408-22. doi:10.1016/j.ijmultiphaseflow.2017.11.008.

[31] Forgacs O, Mason S. Particle motions in sheared suspensions: x. orbits of flexible threadlike particles. J Colloid Sci 1959;14(5):473-91.

[32] Forgacs O, Mason S. Particle motions in sheared suspensions: ix. spin and deformation of threadlike particles. J Colloid Sci 1959;14(5):457-72.

[33] Qi D. Direct simulations of flexible cylindrical fiber suspensions in finite reynolds number flows. J Chem Phys 2006;125:114901-10.

[34] Jeffery G. The motion of ellipsoidal particles immersed in a viscous fluid. Proc R London Ser A 1922;102:161.

[35] Wiggins CH, Riveline D, Ott A, Goldstein RE. Trapping and wiggling: elastohydrodynamics of driven microfilaments. Biophys J 1998;74(2):1043-60. 